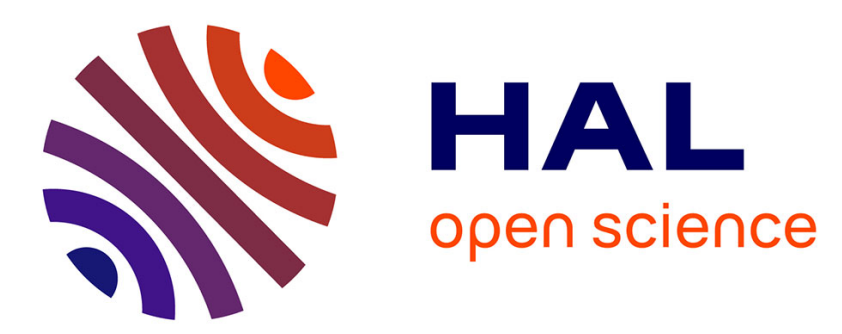

\title{
Scaling and dimensional analysis of acoustic streaming jets
}

B Moudjed, V Botton, D Henry, H Ben Hadid, J.-P Garandet

\section{To cite this version:}

B Moudjed, V Botton, D Henry, H Ben Hadid, J.-P Garandet. Scaling and dimensional analysis of acoustic streaming jets. Physics of Fluids, 2014, 26 (9), pp.093602. 10.1063/1.4895518 . hal$00923712 \mathrm{v} 4$

\section{HAL Id: hal-00923712 \\ https://hal.science/hal-00923712v4}

Submitted on 22 Sep 2020

HAL is a multi-disciplinary open access archive for the deposit and dissemination of scientific research documents, whether they are published or not. The documents may come from teaching and research institutions in France or abroad, or from public or private research centers.
L'archive ouverte pluridisciplinaire HAL, est destinée au dépôt et à la diffusion de documents scientifiques de niveau recherche, publiés ou non, émanant des établissements d'enseignement et de recherche français ou étrangers, des laboratoires publics ou privés. 


\section{AIP $\left.\right|^{\text {Physics of }}$ Fluids}

\section{Scaling and dimensional analysis of acoustic streaming jets}

B. Moudjed, V. Botton, D. Henry, H. Ben Hadid, and J.-P. Garandet

Citation: Physics of Fluids 26, 093602 (2014); doi: 10.1063/1.4895518

View online: http://dx.doi.org/10.1063/1.4895518

View Table of Contents: http://scitation.aip.org/content/aip/journal/pof2/26/9?ver=pdfcov

Published by the AIP Publishing

\section{Articles you may be interested in}

Parabolized Stability Equations analysis of nonlinear interactions with forced eigenmodes to control subsonic jet instabilities

Phys. Fluids 27, 084106 (2015); 10.1063/1.4928472

Numerical simulation of the three-dimensional screech phenomenon from a circular jet

Phys. Fluids 20, 035101 (2008); 10.1063/1.2844474

Macroscopic analysis of gas-jet wiping: Numerical simulation and experimental approach

Phys. Fluids 18, 042103 (2006); 10.1063/1.2186589

Scaling of turbulent wall pressure fluctuations downstream of a rearward facing step

J. Acoust. Soc. Am. 107, L1 (2000); 10.1121/1.428561

Flowfield characteristics of an aerodynamic acoustic levitator

Phys. Fluids 9, 3300 (1997); 10.1063/1.869444

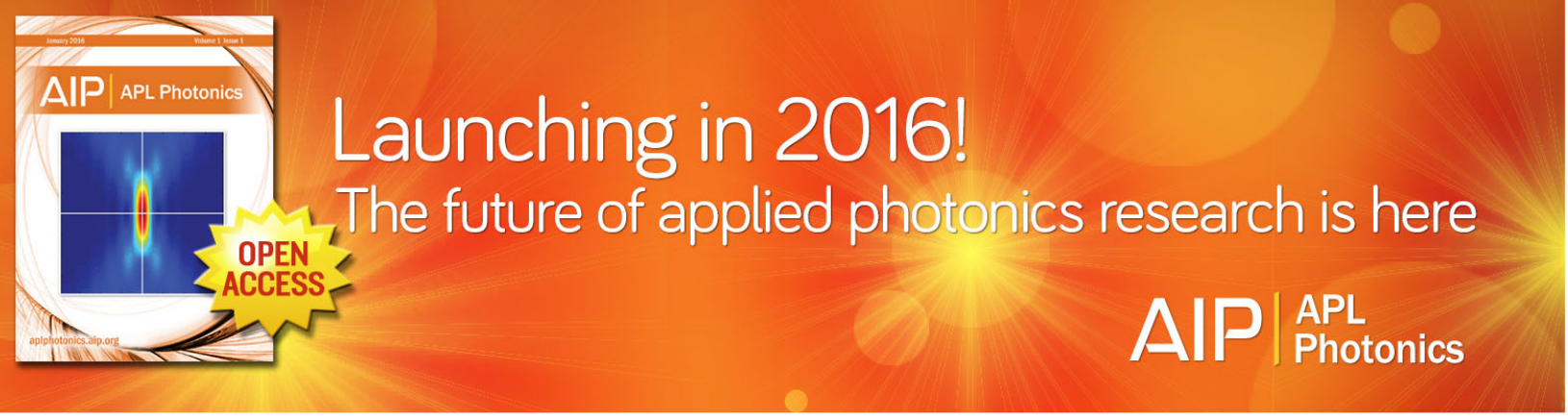




\title{
Scaling and dimensional analysis of acoustic streaming jets
}

\author{
B. Moudjed, ${ }^{1}$ V. Botton, ${ }^{1}$ D. Henry, ${ }^{1}$ H. Ben Hadid, ${ }^{1}$ and J.-P. Garandet ${ }^{2}$ \\ ${ }^{1}$ Laboratoire de Mécanique des Fluides et d'Acoustique, CNRS/Université de Lyon, \\ Ecole Centrale de Lyon/Université Lyon 1/INSA de Lyon, ECL, 36 Avenue Guy de Collongue, \\ 69134 Ecully Cedex, France \\ ${ }^{2} C E A$, Laboratoire d'Instrumentation et d'Expérimentation en Mécanique des Fluides et \\ Thermohydraulique, DEN/DANS/DM2S/STMF/LIEFT, CEA-Saclay, \\ F-91191 Gif-sur-Yvette Cedex, France
}

(Received 20 November 2013; accepted 25 August 2014; published online 18 September 2014)

\begin{abstract}
This paper focuses on acoustic streaming free jets. This is to say that progressive acoustic waves are used to generate a steady flow far from any wall. The derivation of the governing equations under the form of a nonlinear hydrodynamics problem coupled with an acoustic propagation problem is made on the basis of a time scale discrimination approach. This approach is preferred to the usually invoked amplitude perturbations expansion since it is consistent with experimental observations of acoustic streaming flows featuring hydrodynamic nonlinearities and turbulence. Experimental results obtained with a plane transducer in water are also presented together with a review of the former experimental investigations using similar configurations. A comparison of the shape of the acoustic field with the shape of the velocity field shows that diffraction is a key ingredient in the problem though it is rarely accounted for in the literature. A scaling analysis is made and leads to two scaling laws for the typical velocity level in acoustic streaming free jets; these are both observed in our setup and in former studies by other teams. We also perform a dimensional analysis of this problem: a set of seven dimensionless groups is required to describe a typical acoustic experiment. We find that a full similarity is usually not possible between two acoustic streaming experiments featuring different fluids. We then choose to relax the similarity with respect to sound attenuation and to focus on the case of a scaled water experiment representing an acoustic streaming application in liquid metals, in particular, in liquid silicon and in liquid sodium. We show that small acoustic powers can yield relatively high Reynolds numbers and velocity levels; this could be a virtue for heat and mass transfer applications, but a drawback for ultrasonic velocimetry. (C) 2014 AIP Publishing LLC. [http://dx.doi.org/10.1063/1.4895518]
\end{abstract}

\section{INTRODUCTION}

Acoustic streaming flows are steady or quasi-steady flows generated by acoustic waves. Acoustic streaming can be seen as a tool to enhance heat and mass transfer in a number of applications. ${ }^{1-5}$ For instance, several studies show that ultrasounds used during solidification process can improve final material properties. ${ }^{6-12}$ However, it can also unwillingly affect some processes. A typical example is that of Acoustic Doppler Velocimetry (ADV) in which ultrasounds are used to measure velocities in a liquid. This technique is in particular used to investigate flows in opaque liquids, such as liquid metals, ${ }^{13-23}$ muddy waters. However, a recent investigation shows that, depending on the settings used, commercial ADV systems can generate significant acoustic streaming flows, so that a bias is observed in the measurement itself. ${ }^{24}$ This reference study ${ }^{24}$ has been performed in water, but no guideline is given to assess whether acoustic streaming may significantly affect other configurations, e.g., in the case of liquid metals. Acoustic streaming is also present in both existing and developing medical applications based on high intensity ultrasounds, ${ }^{5,25-27}$ but also on 
ultrasounds of lower intensity ${ }^{28}$; the considered liquids are then corporal fluids such as blood or amniotic liquid, the properties of which can significantly differ from those of classical engineering liquids. The discussion developed hereunder might, as a first approximation, apply to these very peculiar cases, but a detailed investigation of them is behind the scope of this paper due to their particular rheology.

This paper is essentially focused on the Eckart streaming ${ }^{29}$ configuration: here, the longitudinal size of the observation domain is far greater than the acoustic wavelength; the acoustic waves are progressive and attenuated waves. Eckart streaming is indeed directly due to sound attenuation in the bulk of the fluid through a Reynolds-stresses-like mechanism: ${ }^{30}$ the fluid motion can be described as an incompressible flow driven by an external volumetric force, which is proportional to the local time-averaged acoustic intensity, $I$, and to the acoustic attenuation coefficient, $\alpha$. An acoustic beam of sufficiently high intensity thus generates a jet flowing in the direction of wave propagation in the region along the beam axis. In closed cavities, a backflow will also occur for the mass conservation law to be verified. In the case considered here, the acoustic beam does not interact with the lateral walls, so that no acoustic boundary layer is present in the problem. Nevertheless, even in cases where acoustic boundary layers exist, it can be shown that in large-scale channels (typically with a size of at least $1 \mathrm{~mm}$ ), the streaming induced by the boundary layers (Rayleigh streaming) is negligible compared with the Eckart streaming. ${ }^{31}$ In fact, the "Rayleigh force" is much stronger than the "Eckart force," but it occurs in very thin layers along the boundaries, so that its effect on the streaming is negligible. ${ }^{32-34}$ This effect would have some importance only in micro-fluidic devices. ${ }^{31}$

Since the pioneering works on the topic, acoustic streaming has very often been presented as a second-order flow. The initially proposed theoretical models leading to the expression of the acoustic streaming force were indeed based on an expansion of each variable in successive approximations. The first order then accounted for the linear acoustic propagation in a quiescent fluid medium, while the second order ruled the acoustic streaming flow. ${ }^{29,32,33,35,36}$ However, several authors conversely underscored that in many experimental investigations and applications, the observed flow was not of second order. ${ }^{30,37,38}$ Experimentalists also observed turbulent acoustic streaming flows, which is not compatible with the assumption of a second order flow, the inertia of which should be negligibly weak. ${ }^{1,30}$ The contrast between the efficiency of acoustic streaming in the potential applications cited above and what could be expected from a second order flow is also questionable. Our contention is that a physical explanation compatible with these experimental observations can be given by considering that the relevant separation of the hydrodynamic problem from the acoustic propagation problem is a time-scales separation rather than an amplitude-scales separation. The frequency of ultrasounds used in liquids is indeed commonly in the Megahertz range or more, which is far greater than the highest frequency component in the considered streaming flows. Note that such a time-scale splitting has already been invoked by Boufermel et al. ${ }^{39}$ but still in the framework of successive approximations regarding variables amplitude, so that the streaming flow remained a second order flow. To our knowledge, no derivation of the acoustic streaming force expression has yet been proposed in the existing literature following this path to get rid of this second order flow assumption.

A number of experimental papers report investigations of acoustic streaming flows. ${ }^{4,37,40-42}$ The results are all described in dimensional variables and most of these experiments are conducted with water, in which a more or less limited amount of particles is added to serve as tracers. They differ in particular by the size and geometry of the water tank and by the values of the ultrasound frequency and intensity. In some of these papers, experimental results are compared to scaling laws giving the dependence of the fluid velocity on the acoustic power. Most authors expect the observed velocities to scale linearly with the acoustic power, but some of them invoke a square root dependence. The assumptions for these expected behaviors to be observed are in addition not explicitly formulated. A common limitation of the literature papers is also that they often do not account for the diffraction of the acoustic field in their analysis. In particular, some of them do not pay any attention to the existence of an acoustic near field, whose extent can be several times the diameter of the sound source for ultrasonic frequencies in liquids. We will show that a scaling analysis, properly performed, can provide interesting information. 
We have finally to note that main measurements of acoustic streaming are done in water experiments, whereas the domains of application involve many other liquids. Dimensional analysis can provide the necessary link. To the best of our knowledge, the dimensional analysis of an acoustic streaming jet has never been reported in any international paper. It is, however, a very efficient tool which, beside the opportunity to extrapolate results from one fluid to another, allows to reduce the investigated parameters space, design model experiments, convert dimensional observations into a more universal non-dimensional form. As our team is interested in applications involving liquid metals, another objective of this paper is thus to investigate the potential of acoustic streaming in some liquid metals.

In the following, the derivation of the acoustic force expression using a time-scale separation approach is given in Sec. II. Some basics of linear acoustics are also briefly recalled since this paper voluntarily takes a hydrodynamics standpoint. The velocity dependence on the acoustic power will be investigated in Sec. III using scaling arguments and taking special care to account for acoustic diffraction. Experimental results from our proper setup, denominated ASTRID (for Acoustic STReaming Investigation Device), ${ }^{38,43}$ as well as from former experimental studies of the literature, ${ }^{4,37,40-42}$ will then be used in Sec. IV to assess the derived characteristic scaling laws. Section $V$ will be devoted to the dimensional analysis of the problem with indications on the way to fulfil the similarity conditions and application of the results to selected liquids. The considered fluids are chosen in typical applications involving high intensity ultrasounds and ADV: water (to which weakly concentrated suspensions and solutions might be assimilated) and liquid metals, namely, liquid silicon and sodium. An important step in this approach is the determination of the sound attenuation coefficient of the considered liquid. These data are actually not always given in usual tables and reference books since its measurement can be difficult in some cases. An additional concern of Sec. V is thus to give guidelines to assess the value of this attenuation coefficient and to properly account for it in a dimensional analysis approach.

\section{PHYSICAL MECHANISMS INVOLVED IN ACOUSTIC STREAMING}

\section{A. An explanation based on time-scales discrimination}

The approach in the following derivation is to split each variable into an acoustic part, varying rapidly, and a streaming motion part, varying very slowly compared to the acoustic part. The acoustic part will be denoted with a subscript $a c$; it is assumed periodical with zero average over one period $T=1 / f$. The streaming motion part will be denoted with a subscript $s$; due to its slow variations, its instantaneous value can be considered equal to its average over one acoustic period, $T$. We start with the continuity and Navier-Stokes equations for a compressible fluid

$$
\begin{gathered}
\frac{\partial \tilde{\rho}}{\partial t}+\operatorname{div}(\tilde{\rho} \tilde{\vec{u}})=0, \\
\tilde{\rho} \frac{d \tilde{\vec{u}}}{d t}=-\overrightarrow{\operatorname{grad}} \tilde{p}+\mu \nabla^{2} \tilde{\vec{u}}+(\eta+\mu) \overrightarrow{\operatorname{grad}}(\operatorname{div} \tilde{\vec{u}}),
\end{gathered}
$$

where the tilde $(\sim)$ denotes any "full" variable before its splitting, and $\rho, \mu$, and $\eta$ are the fluid density, shear viscosity, and bulk viscosity, respectively. In a way similar to the derivation of the Reynolds Averaged Navier-Stokes (RANS) equations, the next step is to introduce the variables decomposition in these equations and compute their average over one period of time. The linear terms will then disappear when they feature an acoustic variable (i.e., with the subscript $a c$ ) or will be substituted with their instantaneous value when they feature a streaming flow variable (namely, those with the subscript $s$ ). The nonlinear terms will disappear when they feature a cross product of an acoustic variable with a streaming flow variable, so that will stay only cross products in which both variables have the same subscript. We can reasonably neglect any variations in viscosity under the effect of the acoustic wave, so that the right-hand side of the averaged Navier-Stokes equation is linear. The left-hand side has terms similar to turbulence Reynolds stresses and thus needs to be 
developed. After computation, the averaged continuity and Navier-Stokes equations are

$$
\begin{gathered}
\frac{\partial \rho_{s}}{\partial t}+\operatorname{div}\left(\overrightarrow{\rho_{a c} \vec{u}_{a c}}\right)+\operatorname{div}\left(\rho_{s} \vec{u}_{s}\right)=0, \\
\rho_{s} \frac{d \vec{u}_{s}}{d t}=-\overrightarrow{\operatorname{grad}} p_{s}+\mu \nabla^{2} \vec{u}_{s}+(\eta+\mu) \overrightarrow{\operatorname{grad}}\left(\operatorname{div} \vec{u}_{s}\right)+\overrightarrow{f_{a c}},
\end{gathered}
$$

where the last term is the acoustic streaming force term, whose $i$ th component in a Cartesian work-frame is

$$
f_{a c, i}=-\operatorname{div}\left(\overline{\rho_{a c} u_{s, i} \vec{u}_{a c}}+\overline{\rho_{a c} u_{a c, i} \vec{u}_{s}}+\overline{\rho_{a c} u_{a c, i} \vec{u}_{a c}}+\rho_{s} \overline{u_{a c, i} \vec{u}_{a c}}\right), i=1,2 \text {, or } 3 .
$$

A simplifying assumption is that terms proportional to $\rho_{a c}$ in Eqs. (4) and (5) can safely be neglected with respect to terms proportional to $\rho_{s}$, in particular in liquids. For example, in the case of water, the isentropic compressibility coefficient is $\chi=5 \times 10^{-10} \mathrm{~Pa}^{-1}$; assuming an acoustic pressure amplitude of $p_{a c}=3 \times 10^{5} \mathrm{~Pa}$, which can be reached in acoustic streaming experiments, the density ratio can be estimated as $\rho_{a c} / \rho_{s}=0.015 \%$. An additional simplification is made by considering that the obtained streaming flow velocities are always far smaller than the velocity of sound ( $c=1480 \mathrm{~m} / \mathrm{s}$ in water), so that the fluid flows at very low Mach number and can be considered as incompressible; in other words, the time and space variations of $\rho_{s}$ are negligible. Eventually, the obtained set of equations for the acoustic streaming flow is

$$
\begin{gathered}
f_{a c, i}=-\rho_{s} \operatorname{div}\left(\overline{u_{a c, i} \vec{u}_{a c}}\right), \\
\operatorname{div} \vec{u}_{s}=0, \\
\rho_{s} \frac{d \vec{u}_{s}}{d t}=-\overrightarrow{\operatorname{grad}} p_{s}+\mu \nabla^{2} \vec{u}_{s}+\overrightarrow{f a c}_{a c}
\end{gathered}
$$

Let us underscore that the time derivative is not here a partial derivative but a particulate derivative, so that the contribution of streaming flow velocity gradients to inertia terms is effectively present in the left-hand side of this formulation. This is an important feature of the present approach, as opposed to the derivations based on small perturbations expansions formerly proposed in the literature. Keep in mind that no hypothesis has been done here on the order of magnitude of the streaming velocities compared to the acoustic velocities.

Let us now consider the case of a plane sinusoidal progressive wave initiated at $x^{\prime}=0$ and propagating with attenuation along the $\boldsymbol{x}$-direction, with an acoustic velocity amplitude of the form:

$$
U_{a c}=\hat{u}_{a c} e^{-\alpha x^{\prime}} .
$$

From Eq. (6), the acoustic streaming force can then be written as

$$
\vec{f}_{a c}=\alpha \rho_{s} \hat{u}_{a c}^{2} e^{-2 \alpha x^{\prime}} \vec{x} .
$$

For such a plane wave, the acoustic intensity can be given as ${ }^{44}$

$$
I=\overline{\rho_{s} c u_{a c}^{2}}=\frac{1}{2} \rho_{s} c \hat{u}_{a c}^{2} e^{-2 \alpha x^{\prime}},
$$

so that we get the following final expression for the force:

$$
\vec{f}_{a c}=\frac{2 \alpha I}{c} \vec{x} .
$$

Such an expression has been previously obtained, but on the basis of a small amplitude expansion approach, which, to our mind, has less physical meaning than the present approach. We have indeed shown that the acoustic streaming flow is ruled by the full Navier-Stokes equations for an incompressible fluid. The additional force term, given by Eq. (12), is the same as that introduced by former authors, ${ }^{30,32,36}$ but here, the hydrodynamic nonlinearity has not to be introduced a posteriori, nor artificially, in the formulation, as underscored above. The flow is thus the solution to a weakly coupled problem between a hydrodynamic sub-problem and an acoustic propagation sub-problem 
with both velocities possibly of the same order of magnitude in the two sub-problems. The coupling is made through the force term (Eq. (12)), which is proportional to the sound amplitude attenuation coefficient. One interest of this approach is also that the fluid has not been assumed to be at rest in this formulation, so that flows driven by other external forces can be accounted for.

The plane wave assumption leading to Eq. (12) and based on Eq. (9) corresponds of course to an idealized situation. In practice, it is reasonable to consider such an assumption to be valid in cases where the acoustic wave propagates in a delimited portion of space, namely, under the form of a beam. In such cases, the plane wave assumption, expressed by Eq. (9), is considered to hold locally, but slow spatial variations of the acoustic velocity amplitude $\hat{u}_{a c}$ have to be considered, in particular, in the transverse direction. The expression "slow spatial variations" can be understood here as variations with a far greater typical length-scale than the acoustic wavelength. As a consequence, the expression of the force must be used in conjunction with a propagation model describing the spatial repartition of the acoustic intensity.

The crudest propagation model is to consider a uniform beam of cylindrical shape, which is to say without any diffraction, nonlinearity nor attenuation ${ }^{3,29,45-49}$; in this case, attenuation is neglected in the propagation problem and is only accounted for through the attenuation coefficient appearing in the acoustic force expression (Eq. (12)). This leads in particular to 1D analytical solutions to the equations of motions. ${ }^{29,45}$ More complex propagation models may account for nonlinear effects, diffraction, and attenuation, ${ }^{37}$ which generally necessitate numerical methods. Former investigations ${ }^{37,38}$ have shown that diffraction is a key ingredient to reproduce the theoretically observed acoustic streaming velocity profiles, and it will be made clear in the following that our opinion is that diffraction should indeed be accounted for; on the other hand, we have shown that a linear propagation model was sufficient to obtain good results in a range of parameters corresponding, at least, to our experimental investigation, ${ }^{38,43}$ which greatly simplifies the handling of the propagation problem. Let us recall that the account of diffraction leads to distinguish a near field and a far field in the acoustic beam emitted by a localized source. ${ }^{44}$ The near field is rather complex to handle since it is strongly varying in space and time, which requires fine spatial discretization in numerical approaches. The far field, on the contrary, is the frame of smooth spatial variations and plane waves are considered as a good approximation in this part of the acoustic field. The choice made in this paper is to restrict the propagation model to linear propagation, but to include diffraction as a key ingredient of the problem.

Prior to the scaling and dimensional analysis of this problem, we think useful to recall some basics of linear acoustics, in particular concerning attenuation and diffraction. It is an appropriate way to introduce the relevant physical parameters of the propagation sub-problem.

\section{B. Basics of acoustics concerning diffraction}

In the following, the wave is assumed monochromatic so that the acoustic field is fully described by the spatial repartition of the amplitude and the phase of the wave. As expected from the linear theory of a piston source in a semi-infinite medium, its structure exhibits a near field and a far field. ${ }^{44}$ Very strong spatial variations occur in the near field, while smoother variations are seen in the far field. The near field zone stretches from the acoustic source to the Fresnel length, $L_{f}$, with

$$
L_{f}=\frac{D_{s}^{2}}{4 \lambda}
$$

where $D_{s}$ is the diameter of the source, and $\lambda=c / f$ is the acoustic wavelength. Note that, at ultrasound frequencies, this near field region can be much longer than the diameter of the source: a source of $3 \mathrm{~cm}$ in diameter emitting $2 \mathrm{MHz}$ ultrasounds in water, for example, has a near field length of $L_{f}=30.4 \mathrm{~cm}$, i.e., ten times the source diameter.

In the far field zone, the acoustic beam diameter is larger than the acoustic source diameter because of diffraction. Indeed, the diameter of the sound beam increases linearly with the distance to the source, so that the sonicated region can be seen as a cone of half-angle $\theta$ such that

$$
\sin \theta=1.22 \frac{\lambda}{D_{s}} .
$$


Let us recall from Eqs. (13) and (14) that small diffraction angle means long near field region, since the Fresnel length to diameter ratio, $L_{f} / D_{s}$, is inversely proportional to the half angle. Ultrasounds wavelengths in water are commonly less than $1 \mathrm{~mm}$, while the sources diameter is of a few dozen of millimeters. The near field zone is thus often far longer than the source diameter and the diffraction angle is small. As a consequence of the linear increase of the beam diameter with the distance to the source, the acoustic intensity rapidly decreases along the longitudinal axis of the beam. Neglecting attenuation, this decrease behaves as $1 / x^{\prime 2}$ in the asymptotically far field.

\section{Basics of acoustics concerning attenuation in liquids}

The acoustic attenuation coefficient inside a liquid, $\alpha$, is very often assumed to have three contributions. ${ }^{50} \mathrm{~A}$ first contribution is connected with the dynamical (or shear) viscosity $\mu$, a second contribution is related to the bulk viscosity $\eta$, and a final contribution takes into account thermal effects. The expression proposed by Nasch et al..$^{50}$ is

$$
N=\frac{\alpha}{f^{2}}=\frac{2 \pi^{2}}{\rho c^{3}}\left(\frac{4}{3} \mu+\eta+\frac{c^{2} \beta^{2} k T}{C_{p}^{2}}\right),
$$

where $f$ is the frequency, $\rho$ is the density, $c$ is the wave velocity, $\beta$ is the thermal expansion coefficient, $k$ is the thermal conductivity, $C_{p}$ is the specific heat, and $T$ is the absolute temperature. The dynamical viscosity $\mu$ and the properties involved in the thermal contribution can generally be obtained for standard liquids with an acceptable accuracy, so that the main difficulty will come from the estimation of the bulk viscosity $\eta$. For example, even for a fluid as much studied as water, various estimations of $\eta$ can be found in the literature, ${ }^{44,51}$ from which different values of the acoustic attenuation coefficient can be obtained. We have summarized the estimations of the different contributions to the acoustic attenuation coefficient for water in each of these cases in Table I (the values of the properties used for water at $20^{\circ} \mathrm{C}$ are $\rho=998.2 \mathrm{Kg} / \mathrm{m}^{3}, \beta=2.07 \times 10^{-4} \mathrm{~K}^{-1}, C_{p}=4180 \mathrm{~J} \mathrm{~K}^{-1} \mathrm{~kg}^{-1}, c=1480 \mathrm{~m} / \mathrm{s}$, $k=0.61 \mathrm{~W} \mathrm{~m}^{-1} \mathrm{~K}^{-1}, \mu=0.001 \mathrm{~Pa}$ ). In Table I, the first column represents a hypothetical water where the bulk viscosity term can be neglected (Stokes hypothesis). We see that for such hypothetical fluid the term connected with thermal effects is small; hence it is commonly neglected, as done here in the three other columns. The term associated with the dynamical viscosity is assumed to be well known, so that the main uncertainty comes from the bulk viscosity which, according to existing literature ${ }^{44,51,52}$ is not at all negligible for water. The acoustic attenuation coefficient can thus vary, for a $2 \mathrm{MHz}$ wave in water, from 0.03 if we neglect the bulk viscosity term (Stokes hypothesis) to $0.083,0.09$, and $0.105 \mathrm{~m}^{-1}$, depending on the value of the bulk viscosity according to the literature. The estimation of $\eta$ given by Kinsler et al. ${ }^{44}$ seems reasonable as it allows finding a value of $N$ close to the measured value, which is reported in the Kaye and Laby online tables ${ }^{53}$ to be $N=\alpha / f^{2}=250$ $\times 10^{-16} \mathrm{~s}^{2} / \mathrm{m}$ for water at $20^{\circ} \mathrm{C}$. We use this measured value in the following. The corresponding

TABLE I. Estimations of the different contributions to the acoustic attenuation coefficient in water.

\begin{tabular}{|c|c|c|c|c|}
\hline Water at $20^{\circ} \mathrm{C}$ & Water (Stokes hypothesis) & Water $^{51}$ & Water $^{52}$ & Water $^{44}$ \\
\hline $\begin{array}{l}\text { Term connected with the bulk } \\
\text { viscosity, } \eta \text { (mPas) }\end{array}$ & 0 & $2.1 \mu=2.1$ & $2.4 \mu=2.4$ & $3 \mu=3$ \\
\hline $\begin{array}{l}\text { Term connected with the dynamical } \\
\text { viscosity, }(4 / 3) \mu(\mathrm{mPas})\end{array}$ & 1.33 & 1.33 & 1.33 & 1.33 \\
\hline $\begin{array}{l}\text { Term connected with thermal } \\
\text { effects ( } \mathrm{mPas})\end{array}$ & $0.96 \times 10^{-3}$ & Neglected & Neglected & Neglected \\
\hline Prefactor $\frac{2 \pi^{2}}{\rho c^{3}}(\mathrm{~s} / \mathrm{mPa} \mathrm{m})$ & $60.63 \times 10^{-16}$ & $60.63 \times 10^{-16}$ & $60.63 \times 10^{-16}$ & $60.63 \times 10^{-16}$ \\
\hline$\alpha / f^{2}\left(\mathrm{~s}^{2} / \mathrm{m}\right)$ & $80.9 \times 10^{-16}$ & $208 \times 10^{-16}$ & $226 \times 10^{-16}$ & $263 \times 10^{-16}$ \\
\hline$\alpha\left(\mathrm{m}^{-1}\right)$ for $f=2 \mathrm{MHz}$ & 0.032 & 0.083 & 0.090 & 0.105 \\
\hline$\alpha\left(\mathrm{m}^{-1}\right)$ for $f=5 \mathrm{MHz}$ & 0.202 & 0.52 & 0.565 & 0.66 \\
\hline
\end{tabular}


values of the attenuation coefficient are typically $\alpha=0.1 \mathrm{~m}^{-1}$ for $f=2 \mathrm{MHz}$ and $\alpha=0.625 \mathrm{~m}^{-1}$ for $f=5 \mathrm{MHz}$.

\section{SCALING ANALYSIS TO GET ORDERS OF MAGNITUDE OF ACOUSTIC STREAMING FLOW VELOCITIES}

In this section, we consider a steady, laminar, acoustic streaming jet in a semi-infinite medium. Under these conditions, the pressure gradient does not play any significant role. The flow is thus governed by a balance between the combined effects of viscosity, inertia, and the acoustic streaming force. We focus successively on the two asymptotic cases of negligible viscous effects and negligible inertia effects.

\section{A. Inertia dominated regime near the origin of the jet}

Let us first consider the acceleration zone near the origin of the jet where inertia plays an important role. The balance between inertial terms and acoustic force can be written, in an order of magnitude sense, on the beam axis

$$
\rho \frac{\partial u^{2}}{\partial x} \propto \frac{2 \alpha I_{a c}}{c} .
$$

The overall power of the beam, $P_{a c}$, is given by

$$
P_{a c}=\int_{0}^{R_{\text {beam }}} I_{a c}(r) 2 \pi r d r
$$

where $R_{\text {beam }}$ is the radius of the acoustic beam. Still from an order of magnitude perspective, we can thus write

$$
I_{a c} \propto \frac{P_{a c}}{\pi R_{b e a m}^{2}} .
$$

From Eq. (16), the typical velocity $u$ at a distance $x$ from the origin of the jet is then expected to follow the following scaling law:

$$
u \propto \sqrt{\frac{2 \alpha}{\rho c} \frac{P_{a c}}{\pi R_{b e a m}^{2}} x .}
$$

At this stage, let us just mention that such a scaling with the acoustic power square root is rarely mentioned in the literature, but has been observed in an experimental work by Mitome. ${ }^{40}$

\section{B. Viscosity dominated regime far from the origin of the jet}

Farther from the origin of the jet, the longitudinal velocity variations can be expected to be weaker so that the occurrence of a flow ruled by the balance between the acoustic force and viscous forces is possible. Assuming that the flow is laminar and nearly one-dimensional, the balance between streaming volumetric forces and viscous forces, can be written in an order of magnitude sense

$$
\frac{2 \alpha I_{a c}}{c} \propto \mu \frac{u}{R_{j e t}^{2}},
$$

in which the acoustic power can again be introduced using Eq. (18), which gives

$$
\frac{2 \alpha}{c} \frac{P_{a c}}{\pi R_{\text {beam }}^{2}} \propto \mu \frac{u}{R_{\text {jet }}^{2}} .
$$

An order of magnitude of the velocity $u$ can be obtained assuming that the jet has nearly the same radius than the beam, i.e., $R_{\text {jet }} \approx R_{\text {beam }}$. Solving equation (21) for $u$ then gives

$$
u \propto 2 \frac{\alpha P_{a c}}{\pi \mu c} .
$$


The proportionality of the measured streaming velocity with the acoustic power is often reported in the literature. In particular, this scaling is claimed to be observed experimentally by Frenkel et al., ${ }^{4}$ Nowicki et al. ${ }^{41,42}$ and Mitome. ${ }^{40}$ A deeper discussion about these observations is proposed in Sec. IV of this paper.

The previous discussion underscores the fact that the cross-section of the jet has not compulsorily the same size as that of the beam, as assumed to derive Eq. (22). In particular, in a semi-infinite domain, two mechanisms can be expected to drive the longitudinal evolution of the jet and the beam: viscous diffusion and diffraction, respectively. The question to answer is thus: why would the jet cross-section scale with the acoustic beam cross section when it is free to do so? Scaling analysis can give clues to answer this question. To start with, the radial enlargement law of a laminar jet by viscous diffusion follows the following scaling law: ${ }^{54,55}$

$$
\frac{\delta R_{\text {diffusion }}}{x}=\sqrt{\frac{v}{u(x) x}}=\operatorname{Re}_{x}^{-1 / 2}
$$

where $x$ is the distance from the origin of the jet, taken as the distance from the upstream wall in our experiment (see Sec. IV). Diffraction leads to the enlargement of the acoustic beam along the $\boldsymbol{x}$-direction in the far field zone (i.e., for $x^{\prime} \geq L_{f}$ ), thus inducing a radial growth of the force-field. In our experiment, this far field zone corresponds approximately to $x=x^{\prime}-L_{f} \geq 0$, as the upstream wall is located close to the Fresnel length. For small angle of diffraction, we can approximate the angle by its tangent; the acoustic beam is then found to increase in radius at the rate

$$
\frac{\delta R_{\text {diffraction }}}{x}=1.22 \frac{\lambda}{D_{s}} .
$$

Comparing diffraction and diffusion enlargement rate, we obtain

$$
\frac{\delta R_{\text {diffraction }}}{\delta R_{\text {diffusion }}}=1.22 \frac{\lambda}{D_{s}} \operatorname{Re}_{x}^{1 / 2} .
$$

This ratio is proportional to the square root of the distance from the wall, $x$. Close to the jet origin, the transverse growth is due to viscous diffusion whereas, far from there, it is driven by diffraction in the acoustic (force) field. Introducing expression (22) in Eq. (25), we find the distance beyond which the jet is governed by diffraction

$$
\frac{x_{\lim }}{L_{f}}=\frac{8 \pi}{1.22^{2}} \frac{\mu^{2} f}{\alpha \rho P_{a c}} .
$$

Note that increasing the acoustic power leads to a decrease in $x_{\text {lim }}$. Equation (26) can be seen as giving an estimate of how long the domain of investigation must be for the order of magnitude given by Eq. (22) to be observed in the acoustic far field.

\section{COMPARISON WITH EXPERIMENTAL DATA}

\section{A. Brief description of our experimental setup}

We have designed a setup denominated ASTRID (for Acoustic STReaming Investigation Device) and dedicated to the characterization of an acoustic streaming flow, which will be briefly presented here; the interested reader is referred to a former publication ${ }^{38}$ for a more detailed description. As shown in Figure 1, a $2 \mathrm{MHz}$ ultrasonic circular plane transducer, with effective diameter $D_{s}=28.5 \mathrm{~mm}$, is placed in an aquarium filled with water. Assuming the sound velocity in water to be $c=1480 \mathrm{~m} / \mathrm{s}$, the Fresnel length value is $L_{f}=274 \mathrm{~mm}$. Two acoustically absorbing plates are introduced. The first plate is put along the wall at the end of the cavity to avoid reflected waves (on the right of the figure). The second plate is drilled with a hole which is covered with a plastic film. This plastic film is seen as a rigid wall for the hydrodynamic problem, while it lets the acoustic waves enter the domain of investigation on the right of the figure. Setting this wall at the position corresponding to the Fresnel length makes it possible to investigate the acoustic streaming flow driven by the acoustic far field in a rectangular cavity. The hole diameter is about twice the transducer diameter. Inner dimensions of the domain of investigation are $16 \mathrm{~cm} \times 18 \mathrm{~cm} \times 47 \mathrm{~cm}$ 


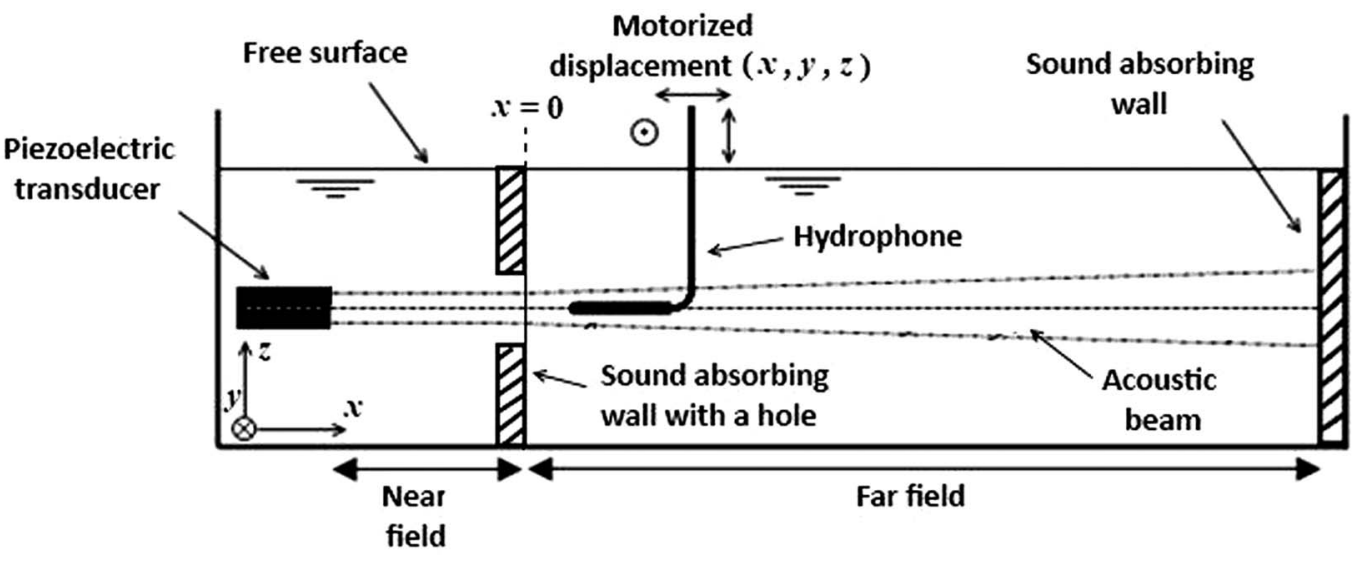

FIG. 1. Experimental configuration (side view); the sound-absorbing wall with the hole is covered with a plastic film to impose a no-slip boundary condition for the hydrodynamic flow. The origin of the coordinates $(\mathrm{x}, \mathrm{y}, \mathrm{z})$ is chosen at the center of this hole. The domain of investigation, situated on the right-hand side of the absorbing wall with a hole $(x>0)$ is $16 \mathrm{~cm}$ deep, $18 \mathrm{~cm}$ wide, and $47 \mathrm{~cm}$ long.

(depth $\times$ width $\times$ length). The acoustic field is characterized via pressure measurements. A threedimensional motorized system is used to move a $1 \mathrm{~mm}$ diameter needle hydrophone from Precision acoustics $^{\mathrm{TM}}$ in order to map the acoustic pressure field in the horizontal middle plane (see Figure 2). We use the Labview ${ }^{\mathrm{TM}}$ software through a PXI unit from National Instruments ${ }^{\mathrm{TM}}$ to supply the transducer via a power-amplifier and a wattmeter, to acquire voltage on hydrophone terminals and to control the motorized system motion. The wattmeter allows us to read the incident electrical power sent to the transducer; this power is regulated to stay constant all along the experiment. The acoustic streaming flow is characterized by Particle Image Velocimetry measurements (PIV) thanks to another independent system. The two characterizations cannot be made simultaneously since the hydrophone and its holder are intrusive; they are removed before carrying out PIV sessions. We formerly took care to check that the measured velocity field is independent of the seeding ${ }^{38}$ (here, $5 \mu \mathrm{m}$ diameter Polyamid Seeding Particles (PSP) particles from Dantec dynamics ${ }^{\mathrm{TM}}$ ).

A typical acoustic pressure amplitude map in the horizontal middle plane of the fluid domain is plotted in Figure 2. To clearly show the near field/far field structure, the absorbing drilled wall has been removed from its location at $x=0$; this location is however represented by a vertical dashed line. The acoustic source is set at $x=-275 \mathrm{~mm}$, i.e., $x \approx-L_{f}$. It has been verified that introducing this wall induces negligible changes in the acoustic field in the investigation area

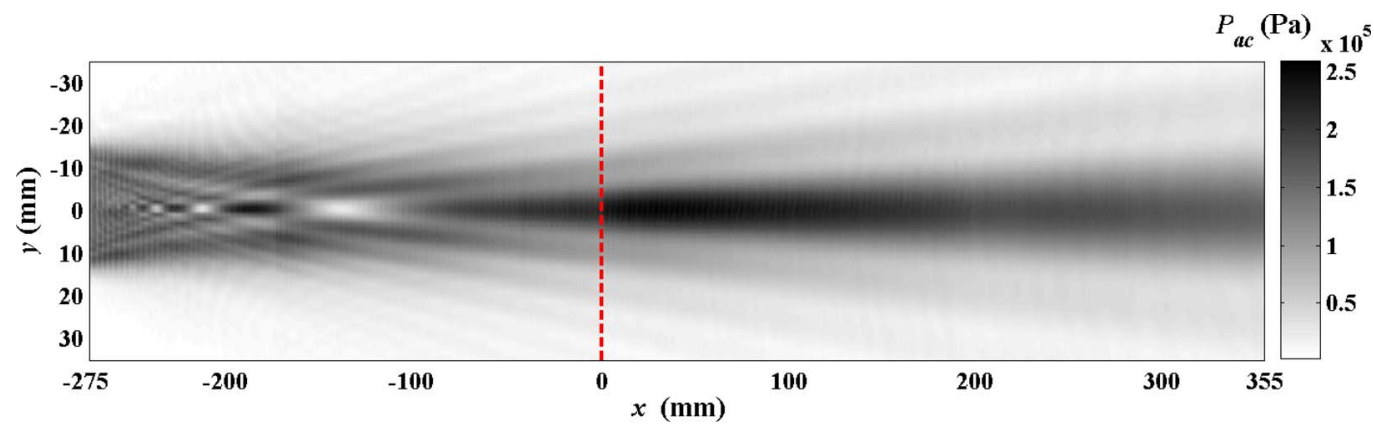

FIG. 2. Experimental map of acoustic pressure amplitude in the horizontal middle plane $(x y)$. The measurement has been made in three separate runs. The acoustic source is situated at $x \approx-L_{f}$. These measurements have been performed without the acoustically transmitting wall otherwise situated at $x=0$ and represented here by a vertical dashed line. The wall has been removed to show the near field/far field structure. Note that the scale is very different on the $y$-axis and on the $x$-axis, so that this figure represents in fact a very elongated region. 
$(x>0)$. The diffraction cone is clearly visible on this picture. Mind that the scale is very different on the $\boldsymbol{y}$-axis and on the $\boldsymbol{x}$-axis of this plot so that the represented region is in fact very elongated.

\section{B. Shape of the jet vs shape of the beam}

Experimental acoustic intensity profiles and axial velocity profiles in the middle horizontal plane are plotted together in Figure 3 at $x=0.5 L_{f}, x=L_{f}$, and $x=1.3 L_{f}$. The profiles are normalized so that they reach the same value at $y / D_{s}=0$; their shape can then be compared more easily.

Clearly, the shape of the three velocity profiles is closely linked to that of the intensity profiles. The oblique dashed lines correspond to the diffraction cone, given by Eq. (14); these lines are seen to roughly correspond to the first local minimum in the acoustic intensity profiles, as expected from the theory. We can notice that this minimum is not a zero. Let us recall that the analytical expression for the intensity profile featuring a Cardinal-Bessel function with zero at this point ${ }^{38}$ is valid in the asymptotically far field only. Here the minimum is non-zero since the measurements are not located far enough from the source for this asymptotic case to be reached. The enlargement of the beam can be observed on the acoustic intensity profiles, plotted with dots: using the oblique dashed lines to define a measure of the beam diameter, we find $24 \mathrm{~mm}$ at $x / L_{f}=0.5,34 \mathrm{~mm}$ at $x / L_{f}=1$ and $40 \mathrm{~mm}$ at $x / L_{f}=1.3$. The transverse velocity profiles plotted as blue lines were obtained for an acoustic power of $2.8 \mathrm{~W}$. Looking very carefully to these profiles, a slight bending in their shape even seems to be present at the level of the secondary local maxima in acoustic intensity. Vertical solid lines represent both the locations where the profiles are taken in the aquarium and the zero value for acoustic and velocity quantities.

This picture clearly supports the assumption made to derive equation (22), namely, that the diameters of the jet and of the beam have similar values. Note that, as in Figure 2, the represented region is very elongated, since $L_{f}=D_{s}{ }^{2} /(4 \lambda) \gg D_{\mathrm{s}}$ in our experiment. The results are also consistent with the scaling analysis of Sec. III, since Eq. (26) predicts that the jet shape could follow the beam shape after a few centimeters only: here $x_{\text {lim }} / L_{f}=0.13$, be $x_{\text {lim }}=3.7 \mathrm{~cm}$. This behavior shows that accounting for diffraction is a key ingredient in the modeling of acoustic streaming for this configuration. Specific mention must be made of the work by Kamakura et al. ${ }^{37}$ which, for the near field region, shows similar results concerning the correlation of velocity and acoustic intensity profiles.

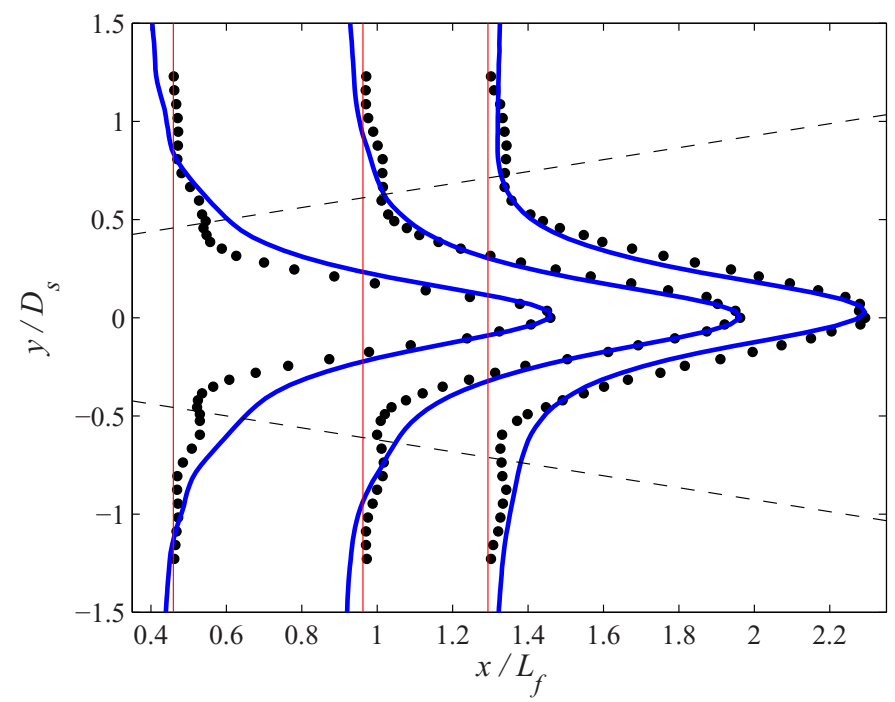

FIG. 3. Experimental profiles of normalized acoustic intensity (dots) and normalized axial velocity (solid black lines) along the $y$ axis at $x=0.5 L_{f}, x=L_{f}$ and $x=1.3 L_{f}$ for an acoustic power of $2.8 \mathrm{~W}$. The two oblique dashed lines correspond to the diffraction cone (Eq. (14)). Note that, since $L_{f}=D_{\mathrm{s}}{ }^{2} /(4 \lambda) \gg D_{s}$ in our experiment, the scale is very different on the $y$-axis and on the $x$-axis, so that this figure represents in fact a very elongated region. 


\section{Confrontation of scaling arguments with present and former experimental data}

Previous velocity measurements of acoustic streaming driven by plane ultrasonic sources in large cavities differ in particular by the size of the source and of the set-up and by the frequency of the ultrasounds used. . $^{47,40-42}$ Experimental investigations featuring focused transducers ${ }^{56,57}$ have voluntary been ignored to restrict our attention to the case of plane sources. The frequency varies from $2 \mathrm{MHz}$ in the present study to $32 \mathrm{MHz}$ in the experiments by Nowicki et al. ${ }^{41,42}$ As the attenuation coefficient of sound in water is proportional to the squared frequency, its values are expected to vary by more than two decades between these studies. Conversely, the maximum power used in these investigations ranges from $0.6 \mathrm{~mW}$ to $10 \mathrm{~W}$, i.e., on more than three decades. This offers a good opportunity to check the scaling laws given in Sec. III. A summary of the experimental conditions in these five investigations, along with those in our own experiment, is shown in Table II. These investigations all involve circular plane transducers emitting continuous waves. The main experimental parameters are recalled in Table II; the Fresnel length and the estimated attenuation coefficient are also indicated. Comparing the Fresnel length to the length of the tank and the location of the measurement point, it can be inferred that some of these measurements are made in the acoustic near field.

Except in Kamakura et al., ${ }^{37}$ where the study is explicitly focused on the acoustic near-field, diffraction is not discussed in these former papers. The investigation zone is in particular not clearly situated with respect to the near field to far field transition. For instance, Mitome ${ }^{40}$ studies the establishment process of acoustic streaming with a $10 \mathrm{~mm}$ diameter, $5.05 \mathrm{MHz}$ ultrasound transducer. Numerical application of Eq. (13) in this case leads to a Fresnel length value of $85.3 \mathrm{~mm}$. Measurement points are chosen at 20,50, 80, 100, and $200 \mathrm{~mm}$ from the transducer, so both in the near field and in the far field without distinguishing the two areas. Likewise, Nowicki et al. ${ }^{41,42}$ measure velocities on the axis of an $8 \mathrm{~mm}$ diameter transducer with a frequency of $32 \mathrm{MHz}$ up to a distance of $15 \mathrm{~mm}$ from the transducer, whereas the near field length is $346 \mathrm{~mm}$. Their investigation is thus restricted to a very small area in the near-field. Similarly, we can estimate that the quantitative data given by Frenkel et al. ${ }^{4}$ are taken in the initial part of the acoustic near field. Finally, Kamakura et al. ${ }^{37}$ explicitly investigate the flow in the acoustic near field, over the whole Fresnel length, with a particular attention to diffraction effects. As exposed here above, our investigation area, on the contrary, begins at the end of the near field, and its length is equivalent to twice the Fresnel length. Our study can thus be seen as complementary to that of Kamakura et al. ${ }^{37}$

A test of Eq. (19) is plotted in Figure 4: velocities measured along the beam axis in these five studies are plotted as a function of the expression appearing under the radical sign in Eq. (19). The values used for the evaluation of this formula are $\rho=998.2 \mathrm{Kg} / \mathrm{m}^{3}, c=1480 \mathrm{~m} / \mathrm{s}$, and $\alpha=N f^{2}$

TABLE II. Main parameters in former and present experimental investigations.

\begin{tabular}{|c|c|c|c|c|c|}
\hline & Nowicki et al. ${ }^{41,42}$ & Mitome $^{40}$ & Frenkel et al. ${ }^{4}$ & Present study & Kamakura et al. ${ }^{37}$ \\
\hline $\begin{array}{l}\text { Rectangular cavity dimensions } \\
(\text { depth } \times \text { width } \times \text { length })(\mathrm{cm})\end{array}$ & $6.4 \times 6.4 \times 9$ & $36 \times 30 \times 60$ & $25 \times 17 \times 14$ & $18 \times 16 \times 47$ & $\begin{array}{c}\text { Cylinder } \\
4.3 \mathrm{~cm} \text { radius } \\
27 \mathrm{~cm} \text { length }\end{array}$ \\
\hline Fluid & Water & Water & Water & Water & Water \\
\hline Source diameter $D_{s}(\mathrm{~cm})$ & 0.8 & 1 & 2.52 & 2.85 & 1.8 \\
\hline Frequency $f(\mathrm{MHz})$ & 32 & 5.05 & 3 & 2 & 5 \\
\hline $\begin{array}{l}\text { Attenuation } \alpha\left(\mathrm{m}^{-1}\right) \alpha=N f^{2} \\
\text { with } N=25 \times 10^{-15} \mathrm{~m}^{-1} \mathrm{~s}^{2}\end{array}$ & 25.6 & 0.637 & 0.225 & 0.1 & 0.57 \\
\hline$L_{f}(\mathrm{~cm})$ from Eq. $(13)$ & 34.6 & 8.53 & 32 & 27.4 & 27.4 \\
\hline Distance $x^{\prime}$ from the & $0-1.5 \mathrm{~cm}$ & $0-20 \mathrm{~cm}$ & $\sim 3 \mathrm{~cm}$ & $L f-3 L f$ & $0-L f$ \\
\hline $\begin{array}{l}\text { transducer to the measurement } \\
\text { point or area }(\mathrm{cm})\end{array}$ & $0-0.04 L_{f}$ & $0-2.3 L_{f}$ & $\sim 0.09 L_{f}$ & & \\
\hline $\begin{array}{l}\text { Investigated acoustic } \\
\text { power range (assuming 100\% } \\
\text { efficiency) (W) }\end{array}$ & $\begin{array}{c}6 \times 10^{-4}-6 \\
\times 10^{-3} \mathrm{~W}\end{array}$ & $1.2 \times 10^{-2}-1.2 \mathrm{~W}$ & $2-10 \mathrm{~W}$ & $0.7-5.6 \mathrm{~W}$ & $0.68 \mathrm{~W}$ \\
\hline
\end{tabular}




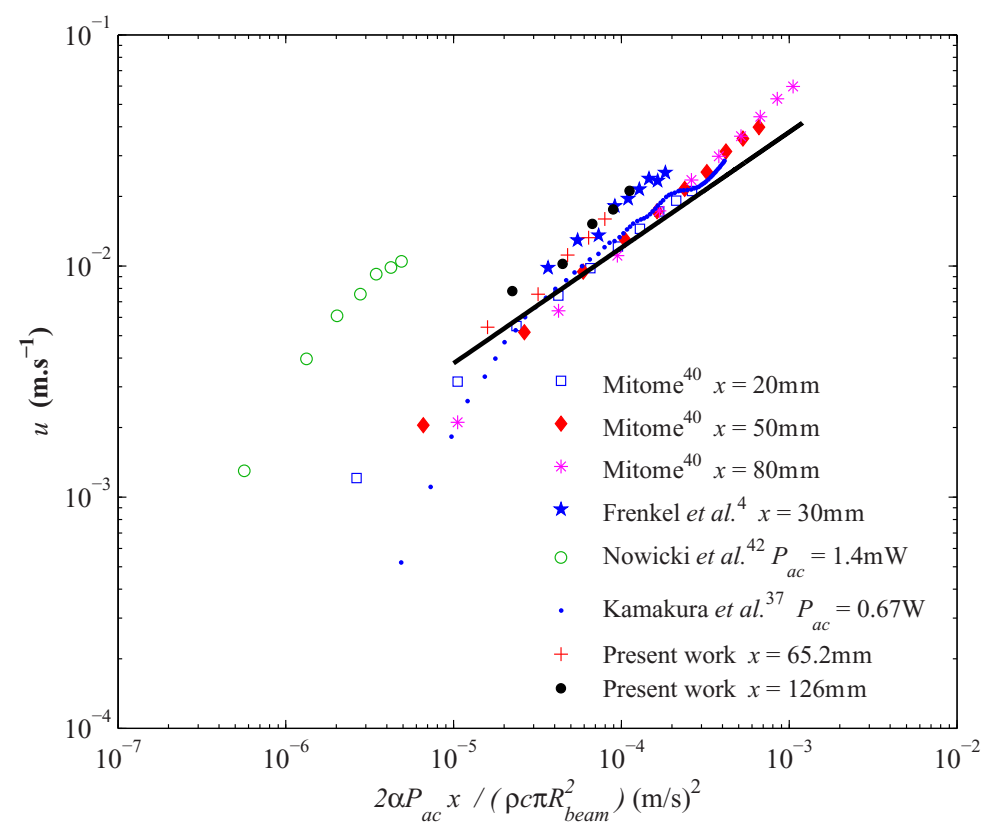

FIG. 4. Plot of experimental velocities to assess the validity of Eq. (19). The velocities measured in our experiment and in several former studies are plotted as a function of the expression appearing under the radical sign in Eq. (19). The black line has a slope of $\frac{1}{2}$ in this $\log -\log$ plot.

with $N=25 \times 10^{-15} \mathrm{~m}^{-1} \mathrm{~s}^{2}$. Concerning $R_{\text {beam }}$, we take $R_{\text {beam }}=R_{s}$, where $R_{s}=D_{s} / 2$ is the source radius, for the near field experiments (Mitome, ${ }^{40}$ Frenkel et al.,${ }^{4}$ Nowicki et al.,${ }^{42}$ and Kamakura et al. $\left.{ }^{37}\right)$, and $R_{\text {beam }}=\left(x+L_{f}\right) \tan \left(\arcsin \left(1.22 \lambda / D_{s}\right)\right)$ for our far field experiment. The data attributed to Mitome ${ }^{40}$ are extracted from Figure 2 of his paper; those attributed to Nowicki et $_{\text {al. }}{ }^{42}$ are the first seven points of the velocity profile plotted in their Figure 4 . The dataset from Kamakura et al. ${ }^{37}$ corresponds to the first $20 \mathrm{~cm}$ of the longitudinal velocity profile plotted in their Figure 6. Note that this last profile is actually obtained numerically; it is reported by these authors to be in agreement with the provided experimental data.

This log-log plot of datasets obtained with different attenuation coefficient, source radius, power, and distance from the wall shows the occurrence of the expected power $\frac{1}{2}$ behavior. Note that Kamakura et al. ${ }^{37}$ and Nowicki et al. ${ }^{42}$ provide data for a fixed value of the power and a varying position, while Mitome, ${ }^{40}$ Frenkel et al.,${ }^{4}$ and our experiment provide data at different values of the power and a fixed value of the position. A steeper slope is observed at low values for Nowicki et $a l .{ }^{42}$ and Kamakura et al. ${ }^{37}$; but these two datasets correspond to different positions in the very near field: typically $x^{\prime} / L_{f}<1 \%$ and $3 \%$ for the data of Nowicki et al. ${ }^{42}$ and Kamakura et al. ${ }^{37}$ respectively. This local inconsistency with the behavior expected from expression (19) may come from the great complexity of the acoustic field in this area, which is not accounted for in the derivation. The oscillations observed at the highest $x$-values in Kamakura's data are due to the strong, but slower, spatial variations of the acoustic intensity along the beam axis near the end of the acoustic near-field. 37,43

It must be underscored that our dataset is the only one on this plot not to be taken in the acoustic near field. The exact location where Frenkel's dataset is measured is not very clearly reported by this author, as well as the source diameter in Nowicki's work. It must also be noticed that Frenkel's data-points appear in this plot to follow a $P_{a c}^{1 / 2}$ behavior, despite the fact that Frenkel presents them to be proportional to $P_{\text {ac }}$ in his Figure 3. Here the rms distance between the regression curve and the data-points is $0.001 \mathrm{~m} \mathrm{~s}^{-1}$, while it would be $0.0028 \mathrm{~m} \mathrm{~s}^{-1}$ assuming proportionality to $P_{a c}$; this confirms that the $P_{a c}{ }^{1 / 2}$ model better describes these data than the $P_{a c}$ model. Frenkel's data are actually taken only 1.4 diameters far from the source, which is very close to the acoustic source. 


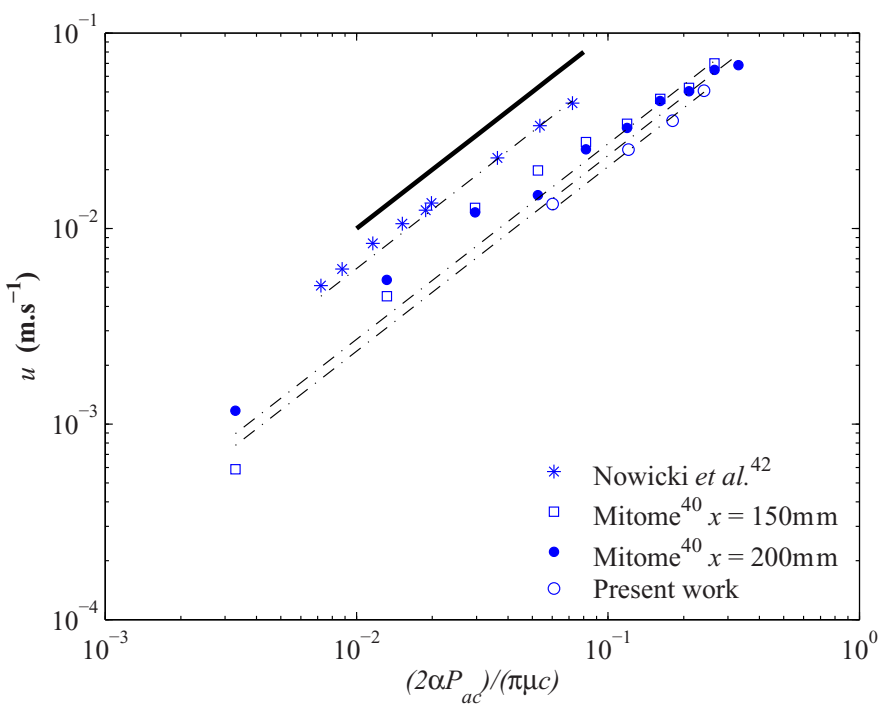

FIG. 5. Plot of experimental velocities to assess the validity of Eq. (22). Markers correspond to experimental measurements obtained at different acoustic powers. The heavy solid line corresponds to Eq. (22); the thin lines are obtained by linear regression to the experimental data.

Mitome $^{40}$ presents velocity data showing proportionality with the acoustic pressure level for three distances from the transducer in Figure 5 of his paper. As the acoustic power is proportional to the squared pressure, this is consistent with Eq. (19), as seen here in Figure 4. Mitome ${ }^{40}$ also provides a plot of the longitudinal velocity profiles (his Figure 2) as a function of the distance to the transducer, which ranges from 0 to $200 \mathrm{~mm}$; it must be stressed that the data used to plot his Figure 5 are all taken at a distance to the transducer smaller than $50 \mathrm{~mm}$. In the following, we use the velocity values from Mitome's Figure 2 at points further from the source to test our second scaling law, namely, Eq. (22).

The test for the scaling given by Eq. (22) is plotted in Figure 5: several datasets are plotted versus the right-hand side of this equation. The experimental dataset attributed to Nowicki et al. ${ }^{42}$ has been extracted from their Figure 2. The values reported here for Mitome ${ }^{40}$ are chosen $150 \mathrm{~mm}$ and $200 \mathrm{~mm}$ far from the acoustic source in their Figure 2. Note that other values, given every $10 \mathrm{mms}$ between these two abscissa, are not reported here for the sake of clarity, but they obviously have the same behavior since this range of abscissa corresponds to a plateau in the longitudinal velocity profiles.

We can consider that the order of magnitude given by Eq. (22) satisfactorily describes the datasets from Nowicki et al. ${ }^{41,42}$ Mitome $^{40}$ and the present team. Indeed the plots show that the variations are nearly linear and the obtained values agree with the scaling assuming a pre-factor a little less than 1. Let us recall that the assumptions for Eq. (22) to hold are the validity of the force expression given by Eq. (12), the occurrence of a viscous force dominated equilibrium, and the equality between the beam and the jet diameters. From Table II, it can be seen that for Mitome's study and our study, the datasets are taken in the far field so that the assumption concerning the diameter of the jet can be converted into a condition on the distance from the wall: it should be greater than the value $x_{\text {lim }}$ given by Eq. (25). The first line of Table III gives the range of values spanned by $x_{l i m}$ in the experiments, computed from the range of involved acoustic power. This is to be compared to the location of the measurement zone also given in Table III. We can see that this assumption is fulfilled in the case of our dataset. It is also fulfilled in the upper range of the acoustic powers investigated by Mitome. ${ }^{40}$

On the other hand, Nowicki et al. ${ }^{41,42}$ operate in the very near field where, as far as we know, even the expression of the acoustic force in this zone is not clearly established from a theoretical standpoint. The linear behavior observed by Nowicki et $a l^{42}$ and the order of magnitude of the measured velocities are both found to be compatible with Eq. (22); at the same time, the data from 
TABLE III. Range of $x_{\text {lim }}$ values estimated from Eq. (26) and distance to the wall in the different experiments.

\begin{tabular}{lcccc}
\hline \hline & Nowicki et al. ${ }^{41,42}$ & Mitome $^{40} x=150 \mathrm{~mm}$ & Mitome $^{40} x=200 \mathrm{~mm}$ & Present study \\
\hline Range of $x_{\text {lim }}(\mathrm{cm})($ Eq. (25)) & $113-1126$ & $1.2-95$ & $1.2-95$ & $1.7-6.6$ \\
Distance to the wall $(\mathrm{cm})$ & 0.6 & 15 & 20 & 26.8 \\
\hline \hline
\end{tabular}

Kamakura et al. ${ }^{42}$ Frenkel et al. ${ }^{4}$ and Nowicki et al. ${ }^{42}$ plotted in Figure 4, all taken in the near field, are also consistent with relation (19). This seems to indicate that the force expression (12), which is used to derive (19) and (22), is at least a good approximation in the near field. This question should be addressed in the future by means of numerical simulations confronted to a dedicated detailed experimental investigation of acoustic streaming flow in the near field..$^{43}$

To sum things up, we have thus presented a scaling analysis giving orders of magnitude for the velocities in acoustic streaming jets. Two scaling laws are proposed depending on whether the momentum balance is dominated by inertia or by viscous forces, namely, Eqs. (19) and (22), respectively. Equation (22) also requires that the beam and the jet have nearly the same transverse dimension. In the acoustic far field, this can be obtained in the case when the transverse growth of the acoustic beam due to diffraction is faster than the viscous diffusion induced transverse growth of the jet. The minimum distance from the origin of the jet for this assumption to hold is given by Eq. (26). As diffraction is a key ingredient in this analysis, our own experimental setup has been designed to account for diffraction: the near and far fields can be separated by an acoustically transmitting wall and the length of the investigation area is several times the Fresnel length. The proposed scaling laws are shown to be globally consistent both with formerly published experimental data and with our proper measurements.

\section{DIMENSIONAL ANALYSIS AND SIMILARITY WITH LIQUID METAL EXPERIMENTS}

In this section, we consider a model experiment in water, designed in similarity with some application featuring another liquid. This is an arbitrary choice since, for example, one could consider a given scale to be a priori chosen for the model; the problem would then be to choose the appropriate model fluid. One of our concerns is indeed to give an estimate of the characteristic size, power, etc., that would have an application, similar to our setup, but operating with liquid metals. The particular cases of liquid silicon and liquid sodium are considered, which makes sense in the general framework of electric energy production and fundamental investigations in planetary and earth sciences. Liquid silicon is involved in crystal growth processes for solar-cells production, in which stirring by acoustic streaming could be of interest. Liquid sodium is used in nuclear plants design ${ }^{13,18}$ and in academic experiments concerning the earth and planetary dynamo effect, applications which often involve ultrasonic velocimetry. ${ }^{14-16}$ Of course, other liquid metals or alloys could be of interest ${ }^{17,21-23,58}$; we will here focus mainly on $\mathrm{Si}$ and $\mathrm{Na}$, which, with their very different melting point and density, are representative of the behaviors that can be met with many metallic liquids. Iron will also be considered, as it is a reference liquid metal which is better documented. This section can also be seen as giving guidelines to assess acoustic streaming flows in any Newtonian fluid.

\section{A. Dimensional analysis}

As a consequence of the former developments, we consider that a good description of an acoustic streaming free jet must necessarily feature both a description of the acoustic propagation problem, including diffraction, and a description of the incompressible fluid flow problem. Let us consider that the aim of the analysis is to investigate the link between the acoustic power $P_{a c}$ of the sound source and an observed typical velocity $U$. If we consider a simple fluid domain of square cross section of side $l$ and length $L$, the hydrodynamic problem features 5 variables: the velocity, $U$, the liquid density and kinematic viscosity, $\rho$ and $v$, and these two dimensions, $l$ and $L$. Five-dimensional variables are also required to describe the acoustic problem: the power and diameter of the plane source, $P_{a c}$ and 
TABLE IV. Variables of the problem, their units and the corresponding dimensionless groups.

\begin{tabular}{lccc}
\hline \hline Dimensional variables & Usual units & Fundamental units & Corresponding dimensionless group \\
\hline$N=\alpha / f^{2}$ & $\mathrm{~m}^{-1} \mathrm{~Hz}^{-2}$ & $\mathrm{~m}^{-1} \mathrm{~s}^{2}$ & $\mathbf{N}=N f^{2} L$ \\
$f$ & $\mathrm{~Hz}$ & $\mathrm{~s}^{-1}$ & $\mathbf{F}=f D_{s}{ }^{2} / v$ \\
$\lambda=c l f$ & $\mathrm{~m}$ & $\mathrm{~m}$ & $\mathbf{S}=1.22 \lambda / D_{s}$ \\
$L, l$, and $D_{s}$ & $\mathrm{~m}$ & $\mathrm{~m}$ & $\mathbf{L}=L / D_{s}, \mathbf{l}=l / D_{s}$ \\
$P_{a c}$ & $\mathrm{~W}$ & $\mathrm{~kg} \mathrm{~m}^{2} \mathrm{~s}^{-3}$ & $\mathbf{P}=P_{a c} \cdot D_{\mathrm{s}} /\left(\rho \cdot v^{3}\right)$ \\
$U$ & $\mathrm{~m} \mathrm{~s}^{-1}$ & $\mathrm{~m} \mathrm{~s}^{-1}$ & $\mathbf{U}=U \cdot D_{s} / v$ \\
$v$ & $\mathrm{~m}^{2} \mathrm{~s}^{-1}$ & $\mathrm{~m}^{2} \mathrm{~s}^{-1}$ & $\ldots$ \\
$\rho$ & $\mathrm{kg} \mathrm{m}^{-3}$ & $\mathrm{~kg} \mathrm{~m}^{-3}$ & $\ldots$ \\
\hline \hline
\end{tabular}

$D_{s}$, the sound attenuation coefficient $\alpha$, as well as the frequency and celerity of the wave, $f$ and $c$. As discussed in Sec. III, in Newtonian fluids, the attenuation is known to be proportional to the square of the frequency. ${ }^{44,50}$ We thus replace $\alpha$ in the list of parameters by $N=\alpha / f^{2}$, which is a frequency independent physical property of the material. Relying on the Vaschy-Buckingham theorem, and choosing $D_{s}, D_{s}{ }^{2} / \nu$, and $\rho D_{\mathrm{s}}{ }^{3}$ as characteristic scales for length, time, and mass, respectively, we define 7 dimensionless groups, which are required to fully characterize this problem. They are listed in Table IV.

The dimensionless form of the governing equations with this set of dimensionless parameters is given in the Appendix of this paper.

\section{B. Similarity conditions with liquid metal experiments}

Let us denote $\Sigma$ the scale of the test case (i.e., the water experiment) with respect to the real case (liquid metal experiments). In addition, the parameters referring to the test case and to the real case will be denoted with a subscript test and real, respectively. With these notations, the scale $\Sigma$ is thus defined by

$$
\Sigma=\frac{\mathrm{L}_{\text {test }}}{\mathrm{L}_{\text {real }}}
$$

Finally, the ratio between the value of any parameter $m$ in the test case and in the real case will be denoted as $R_{m}=m_{\text {test }} / m_{\text {real }}$. The similarity analysis can be first considered from the linear acoustic problem standpoint. The spatial variations of the acoustic field are characterized by the three dimensionless groups $\mathbf{N}, \mathbf{F}$, and $\mathbf{S}$; the following set of equations has thus to be verified:

$$
\left\{\begin{array} { l } 
{ \mathbf { N } _ { \text { test } } / \mathbf { N } _ { \text { real } } = 1 , } \\
{ \mathbf { F } _ { \text { test } } / \mathbf { F } _ { \text { real } } = 1 , } \\
{ \mathbf { S } _ { \text { test } } / \mathbf { S } _ { \text { real } } = 1 , }
\end{array} \quad \text { or } \quad \left\{\begin{array}{l}
R_{N} R_{f}^{2} \Sigma=1, \\
R_{f} \Sigma^{2} R_{v}^{-1}=1, \\
R_{c} R_{f}^{-1} \Sigma^{-1}=1 .
\end{array}\right.\right.
$$

As we consider the fluid couple to be fixed here, for given ambient conditions, $R_{v}, R_{c}$, and $R_{N}$ are fixed since they depend on the fluid properties only, while $\Sigma$ and $R_{f}$ relate to the experimental configurations and must be chosen for the two cases to be in similarity. Unfortunately this three equation system with two unknowns is over-determined and usually has no solution. A full similarity is thus generally not possible and a choice must be made to limit the acoustic similarity to two dimensionless groups, at the expense of the third one. Note that, in the general framework of fluid dynamics, it is rare to be able to establish a full similarity between two hydrodynamic problems (for example, see the case of free surface hydraulics, in which a similarity in terms of the Reynolds number and the Froude number is not possible in a water model experiment ${ }^{59}$ ).

As discussed above, we consider that diffraction is a key element in the modeling of acoustic streaming flows, which means that the dimensionless group $\mathbf{S}$ has to be kept in the problem for- 
mulation. We choose to relax the similarity condition concerning the account of attenuation in the propagation problem and then drop the dimensionless group $\mathbf{N}$. This does not mean that attenuation is neglected in the problem, but rather that we accept that the attenuation occurring in the test case is not quantitatively representative of the attenuation in the real case. The system of equations (28) is then reduced to its second and third lines and has the following solutions for the scale and frequency ratios:

$$
\Sigma=\frac{R_{v}}{R_{c}}
$$

and

$$
R_{f}=\frac{R_{c}^{2}}{R_{v}} .
$$

We can now compute the ratio of the dimensionless group $\mathbf{N}$ for the test case and the reality as a function of the fluids properties,

$$
\frac{\mathbf{N}_{\text {test }}}{\mathbf{N}_{\text {real }}}=\frac{R_{N} R_{c}^{3}}{R_{v}} .
$$

As discussed above, this ratio is not equal to unity in the general case. The difference with respect to unity can be used to quantify how restrictive was the choice made when we decided to relax the attenuation similarity in the propagation problem.

Attenuation, however, plays a very important role in our problem, as it is, inside the force term, the link between acoustics and hydrodynamics. To account for this link, while the similarity for the dimensionless group $\mathbf{N}$ has been dropped out, we can replace the dimensionless power $\mathbf{P}$ by the group NP. The meaning of this substitution is that, in view of streaming issues, what matters most for the hydrodynamics problem is the acoustic streaming force, which is proportional to the attenuation coefficient times the acoustic power (global standpoint) or the acoustic intensity (local standpoint). This is particularly clear in the dimensionless form of the governing equations given in the Appendix of this paper (see Eqs. (A2) and (A6)-(A8)). A meaningful similarity is then obtained for $\mathbf{N}_{\text {test }} \mathbf{P}_{\text {test }}=\mathbf{N}_{\text {real }} \mathbf{P}_{\text {real }}$, which gives

$$
R_{P_{a c}}=\frac{R_{\rho} R_{v}^{3}}{R_{N} R_{c}^{2}} .
$$

Finally, the Reynolds similarity can be written as $\mathbf{U}_{\text {test }}=\mathbf{U}_{\text {real }}$, which gives the following ratio between test case velocity and real case velocity:

$$
R_{U}=\frac{R_{v}}{\Sigma}=R_{c}
$$

We can notice that this relation is equivalent to a Mach similarity in which the Mach number is defined as $\mathbf{M}=U / c$. We preferably use the Reynolds number to the Mach number since the generated flow is incompressible and its transport properties (Nusselt number, Sherwood number, etc.) are expected to be directly correlated to the Reynolds number.

\section{Estimation of the acoustic attenuation coefficient for a selection of liquids}

One of the difficulties when extrapolating experimental results obtained in water to other fluids is to find the properties of the concerned fluids. In particular, the acoustic attenuation coefficient is often poorly documented. For liquid iron, silicon, and sodium, the values of the properties that are useful for its estimation from Eq. (15) are given in Table V. The values for iron have been obtained from Nasch et al. ${ }^{50}$ the values for silicon from different authors, ${ }^{60-64}$ and the values for sodium from Sobolev. ${ }^{65}$ We see that the main values are available, except the bulk viscosity for silicon and sodium. Note, however, that an expression is proposed for iron, $\eta=(3 / 5) \mu$, which will be used as a first approximation for silicon.

The different contributions to the acoustic attenuation coefficient for iron, silicon and sodium are given in Table VI. We see that, for these liquid metals, the thermal contribution is very large and, in 
TABLE V. Physical property values useful for the estimation of the acoustic attenuation coefficient in liquid iron, silicon, and sodium.

\begin{tabular}{lccc}
\hline \hline Property & Iron at $1809 \mathrm{~K}^{50}$ & Silicon at $1700 \mathrm{~K}$ & Sodium at $393 \mathrm{~K}^{65}$ \\
\hline Specific heat $C_{P}\left(\mathrm{~J} \mathrm{~K}^{-1} \mathrm{~kg}^{-1}\right)$ & 748.5 & $1000^{64}$ & 1374 \\
Thermal conductivity $k\left(\mathrm{~W} \mathrm{~m}^{-1} \mathrm{~K}^{-1}\right)$ & 32.2 & $60^{60}$ & 85.5 \\
Thermal expansion $\beta\left(\mathrm{K}^{-1}\right)$ & $0.82 \times 10^{-4}$ & $1.1 \times 10^{-4} 60$ & $2.55 \times 10^{-4}$ \\
Sound celerity $c(\mathrm{~m} / \mathrm{s})$ & 3900 & $3900^{61}$ & 2514 \\
Density $\rho\left(\mathrm{Kg} / \mathrm{m}^{3}\right)$ & 6980 & $2500^{60}$ & 922 \\
$\mu(\mathrm{mPa} \mathrm{s})$ & 5 & $0.8^{62}$ & 0.62 \\
$\eta(\mathrm{mPas})$ & $(3 / 5) . \mu=3$ & $\cdots$ & $\cdots$ \\
\hline \hline
\end{tabular}

any case, much stronger than the contribution connected with the dynamical viscosity, particularly for silicon where the ratio is close to 17. Concerning the bulk viscosity, we see that it is of the same order as the dynamical viscosity for iron (factor 0.6), whereas it was a little larger (factor 3 ) for water. For silicon, in view of the small value of $\mu$, the contribution of the bulk viscosity can be expected to remain small and, in any case, far smaller than the thermal contribution. This contribution could have been neglected, but we chose to deduce it from the relationship between $\eta$ and $\mu$ used for iron. The values of the acoustic attenuation coefficient thus obtained for silicon ( $\alpha=0.01$ for a $2 \mathrm{MHz}$ wave) are larger than those for iron $(\alpha=0.004$ for a $2 \mathrm{MHz}$ wave) due to the larger thermal contribution, but they are small compared to the values found for water, about ten times smaller. For sodium, the thermal contribution is still dominant, and we choose to approximate the bulk viscosity by $\eta=1.25 \mu$, so that the value of $\alpha / f^{2}$ is close to the measured value found in the Kaye and Laby online tables ${ }^{53}$ for sodium at $383 \mathrm{~K}\left(\alpha / f^{2}=120 \times 10^{-16} \mathrm{~s}^{2} / \mathrm{m}\right)$. The values of the acoustic attenuation coefficient obtained for sodium are then significantly larger than for the two other liquid metals, and closer to the values for water (about half the values for water).

\section{Implications for experimental works}

Table VII gives the numerical evaluation of equations (29)-(33) in the case of liquid silicon and sodium a few degrees above their respective melting points. As liquid metals exhibit both lower viscosities and higher sound celerities than water, a water model should be bigger than the setup it represents; the scale given in the first column of Table VII shows that the model must be more than eight times bigger in the case of silicon, while it is nearly twice bigger in the case of sodium. Note that this means that a water experiment must be 550 times bigger in volume than its silicon

TABLE VI. Estimations of the acoustic attenuation coefficient in liquid iron, silicon, and sodium close above the melting temperature.

\begin{tabular}{lccc}
\hline \hline $\begin{array}{l}\text { About } 2{ }^{\circ} \mathrm{C} \text { above melting } \\
\text { temperature }\end{array}$ & Iron $^{50}$ & Silicon & Sodium \\
\hline $\begin{array}{l}\text { Term connected with the bulk } \\
\text { viscosity, } \eta \text { (mPa s) }\end{array}$ & $0.6 \mu=3$ & Approximated by $0.6 \mu=0.48$ & Approximated by $1.25 \mu=0.77$ \\
$\begin{array}{l}\text { Term connected with the } \\
\text { dynamical viscosity, }(4 / 3) \mu(\mathrm{mPa} \mathrm{s})\end{array}$ & 6.66 & 1.07 & 0.825 \\
$\begin{array}{l}\text { Term connected with thermal } \\
\text { effects }(\mathrm{mPa})\end{array}$ & 10.62 & 18.15 & 7.3 \\
Prefactor $\frac{2 \pi^{2}}{\rho c^{3}}(\mathrm{~s} / \mathrm{mPa} \mathrm{m})$ & $0.476 \times 10^{-16}$ & $1.33 \times 10^{-16}$ & \\
$\alpha / f^{2}\left(\mathrm{~s}^{2} / \mathrm{m}\right)$ & $9.65 \times 10^{-16}$ & $26.2 \times 10^{-16}$ & $13.5 \times 10^{-16}$ \\
$\alpha\left(\mathrm{m}^{-1}\right)$ for $f=2 \mathrm{MHz}$ & 0.004 & 0.01 & $120 \times 10^{-16}$ \\
$\alpha\left(\mathrm{m}^{-1}\right)$ for $f=5 \mathrm{MHz}$ & 0.024 & 0.065 & 0.048 \\
\hline \hline
\end{tabular}


TABLE VII. Scale and ratio of the main parameters for a model experiment in water in similarity with test cases featuring silicon and sodium.

\begin{tabular}{lccccc}
\hline \hline & $\begin{array}{c}\text { Scale } \boldsymbol{\Sigma} \\
(\text { Eq. (29)) }\end{array}$ & $\begin{array}{c}f_{\text {test }} / f_{\text {real }} \\
(\text { Eq. (30)) }\end{array}$ & $\begin{array}{c}\mathbf{N}_{\text {test }} / \mathbf{N}_{\text {real }} \\
\text { (Eq. (31)) }\end{array}$ & $\begin{array}{c}P_{a c, \text { test }} / P_{a c, \text { real }} \\
\text { (Eq. (32)) }\end{array}$ & $\begin{array}{r}U_{\text {test }} / U_{\text {real }} \\
(\text { Eq. (33)) }\end{array}$ \\
\hline Silicon $(1750 \mathrm{~K})$ & 8.2 & 0.046 & 0.17 & 8.9 & 0.38 \\
Sodium (393 K) & 2.5 & 0.23 & 0.28 & 4.9 & 0.59 \\
\hline \hline
\end{tabular}

counterpart, which might be prohibitive, and only 6.8 times bigger than its sodium counterpart. For the same reasons, the frequency used in the water model should always be smaller than in the liquid metal application; while the ratio is between one fourth and one fifth for liquid sodium, it is less than 1 over 20 for liquid silicon. Let us recall that the ratio $\mathbf{N}_{\text {test }} / \mathbf{N}_{\text {real }}$ in the third column should be one for the similarity in term of attenuation to be respected. Here again the case of the sodium-water similarity is the most favorable. A water model cannot accurately reproduce a problem with liquid silicon in which attenuation would significantly influence the spatial repartition of acoustic energy.

The power-ratio given in the fourth column can be seen as the ratio in acoustic powers to reach the same Reynolds number in water and in the liquid metal. It is greater than one in both cases; however, it is nearly identical to the scale for the silicon/water couple, while it is twice the scale for the sodium/water couple. Let us remark that this ratio is very sensitive to the ratio of the viscosities in the two fluids, which appears with a power three in Eq. (32). We can thus see that the required power to reach a given Reynolds number in liquid silicon is nearly one tenth of what it is in water, a quite remarkable feature in terms of potential applications. In many applications, the Reynolds number is indeed the appropriate dimensionless number to characterize the effect of convection, since heat and mass transfer properties of the flow (characterized, for example, by the Nusselt and Sherwood numbers, respectively) are directly correlated to it. In the case of velocimetry, what matters is rather the dimensional velocity potentially induced by the acoustic streaming. In our setup $\left(D_{s}=28.5 \mathrm{~mm}\right.$, $f=2 \mathrm{MHz}$ ), $1 \mathrm{~W}$ of acoustic power produces a streaming velocity on the order of $1 \mathrm{~cm} / \mathrm{s}$ in water (which can be retrieved as an application of Eq. (22)). Using this starting point, the second line of Table VII shows that, in liquid sodium, a plane transducer of diameter $12 \mathrm{~mm}$ operating at $8.6 \mathrm{MHz}$ would induce velocities on the order of $1.7 \mathrm{~cm} / \mathrm{s}$ with an acoustic power of only $200 \mathrm{~mW}$. Because of the scale $\boldsymbol{\Sigma}$, we can also say that these velocities would be obtained after a smaller distance from the source. As mentioned earlier, this numerical application makes us think that it should be taken care of acoustic streaming side-effects in ADV measurements.

\section{CONCLUSION}

This paper focuses on acoustic streaming free jets. This is to say that progressive acoustic waves are used to generate a steady flow in the bulk of a liquid. In particular, no acoustic boundary layer is present in the problem since the acoustic beam does not interact with lateral walls.

The derivation of the governing equations under the form of a nonlinear hydrodynamics problem coupled with an acoustic propagation problem is based on a time scale discrimination approach. This approach is preferred to the usually invoked amplitude perturbations expansion. Though the obtained expression for the source term in the Navier-Stokes equations is the same, this original approach provides a consistent framework for the experimental observations of acoustic streaming flows that can feature hydrodynamic nonlinearity and turbulence.

A scaling analysis is performed on the basis of these equations of motion including the acoustic force. Two scaling laws are proposed for the velocity level observed on the acoustic beam axis, depending on the most significant term considered as balancing the acoustic streaming force in the steady state momentum budget. Considering inertia, which corresponds to the acceleration zone near the origin of the jet, the velocity is expected to scale as the square root of both the acoustic power and the distance from the wall, $x$. When considering viscous effects, the velocity is expected to vary linearly with the power and to be nearly independent of $x$. In the acoustic far field, this regime is 
expected if the jet thickening by viscous diffusion is slower than the beam thickening by diffraction, which should be observed in long enough apparatus.

Experimental results are also presented together with a review of former experimental investigations concerning the power-to-velocity relationship for plane ultrasonic transducers in water. Our experimental study, focused on the flow in the far field, is complementary to that of Kamakura et al.,${ }^{37}$ which was restricted to the near field. Our apparatus is well designed to observe the correlation between the acoustic intensity and the velocity fields of the acoustic streaming driven jet under the effect of acoustic diffraction. This is illustrated here by presenting normalized transverse velocity profiles plotted on the same figure as normalized acoustic intensity profiles. This comparison of the acoustic field and the velocity field in our water experiment thus confirms that diffraction is a key ingredient in the problem. This is an important conclusion since this aspect is rarely accounted for in the literature. The literature review is also used as a test for the two scaling laws which are both observed in our setup and in former studies by other teams. The observation of the same scaling laws in the near acoustic field seems to indicate that the expression of the force established in the acoustic far field, namely, Eq. (12), is also valid, at least in an order of magnitude sense, in the near field. This issue should be investigated by numerical means and dedicated experiments in the near future.

Experimental investigations related to acoustic streaming in the literature, including our own study, are yet limited to experiments in water. A number of applications, however, make use of other Newtonian fluids, and in particular liquid metals. We thus propose a dimensional analysis of this problem. As diffraction has been shown to be a key ingredient in the problem, the dimensional analysis of this problem must include the acoustic parameters (celerity of sound, attenuation coefficient, and diameter of the sound source), the geometrical parameters (aspect ratios), and the incompressible fluid flow parameters (density and viscosity). A set of seven dimensionless groups is then required to describe a typical acoustic experiment in a parallelepipedic aquarium of square cross-section; two of these are dedicated to the description of the geometry, so that for a model experiment at any scale, five dimensionless groups are to be considered. We show that a full similarity is usually not possible and that the similarity condition must be relaxed for one parameter. We show that it is preferable to relax the similarity with respect to the sound attenuation, since we consider experimental configurations a priori featuring liquids and at relatively low frequencies, in which neglecting attenuation at first order when computing the acoustic field would not be too critical. The scale of the model experiment is imposed by the properties of the two considered fluids; it is given by Eq. (29). The ratios in frequency, power, and velocity between the model experiment and the real case are given by Eqs. (30), (32), and (33), respectively. Equation (31) is itself a quantification of how restrictive is the relaxation of the similarity with respect to attenuation.

When willing to apply relations (29)-(33) to real cases, the need of data becomes pregnant. In particular, the sound attenuation coefficient is not always known. This is in particular the case for melt silicon and sodium, which we have chosen to illustrate the case of crystal growth applications, nuclear applications, and earth and planetary science experiments. It was, however, possible to rely on the knowledge of other physical properties to compute an estimate of the attenuation coefficient. Concerning the similarity analysis, in the case of liquid silicon, the striking result is that reaching a Reynolds number within a silicon setup requires only one tenth of the power needed to reach the same Reynolds number in the water model experiment. Such a flow would, however, be obtained in a far smaller sonicated volume. This part of the paper has been written with enough details to serve as guidelines for possible other studies with different liquids. In the case of liquid sodium, we show that our experimental setup may be not far from being in similarity with ultrasonic velocimetry apparatus; a numerical application shows that small powers can yield significant acoustic streaming in liquid sodium, which could yield a bias in velocimetry measurements and should better be accounted for in the future.

\section{ACKNOWLEDGMENTS}

Funding for this project was provided by a grant, including a doctoral fellowship for Brahim Moudjed, from la Région Rhône Alpes. 


\section{APPENDIX: GOVERNING EQUATIONS AND SCALING LAWS IN DIMENSIONLESS FORM}

With the set of dimensionless parameters defined in Sec. V, the governing equations (7) and (8) become, respectively,

$$
\operatorname{div} \mathbf{u}=0 \text { and } d \mathbf{u} / d \mathbf{t}=-\vec{\nabla} \mathbf{p}+\vec{\nabla}^{2} \mathbf{u}+\mathbf{F} \overrightarrow{\mathbf{x}}
$$

with $\mathbf{t}=t /\left(D_{s}{ }^{2} / \nu\right)$ and $\mathbf{p}=p /\left(\rho v^{2} / D_{s}{ }^{2}\right)$. The force expressed by (12) becomes:

$$
\overrightarrow{\mathbf{F}}=\mathbf{F} \overrightarrow{\mathbf{x}}=2.44 \frac{\mathbf{N} . \mathbf{P}}{\text { S.F.L }} \mathbf{G}(\mathbf{x}, \mathbf{y}, \mathbf{z}) \overrightarrow{\mathbf{x}},
$$

where $\mathbf{G}$ is the dimensionless spatial repartition of the acoustic intensity defined by

$$
\mathbf{G}=\frac{I \cdot D_{s}^{2}}{P_{a c}} .
$$

Expressions (13) and (14) also give

$$
\begin{gathered}
\mathbf{L}_{\mathbf{f}}=\frac{L_{f}}{D_{s}}=\frac{1.22}{4 \mathbf{S}}, \\
\sin \theta=\mathbf{S} .
\end{gathered}
$$

Similarly, the scaling relations of Sec. III, respectively, Eqs. (19), (22), and (26), can be written with dimensionless variables only as

$$
\begin{gathered}
\mathbf{U} \propto \sqrt{\frac{8 \times 1.22}{\pi} \frac{\mathbf{N . P}}{\mathbf{S . F}}\left(\frac{x}{L}\right),} \\
\mathbf{U} \propto \frac{2.44}{\pi} \frac{\mathbf{N . P}}{\mathbf{L . S . F}}, \\
\frac{x_{\lim }}{L_{f}}=\frac{8 \pi}{1.22^{2}} \frac{\mathbf{F . L}}{\mathbf{N . P}} .
\end{gathered}
$$

${ }^{1}$ M. C. Schenker, M. J. B. M. Pourquié, D. G. Eskin, and B. J. Boersma, "PIV quantification of the flow induced by an ultrasonic horn and numerical modeling of the flow and related processing times," Ultrason. Sonochem. 20, 502 (2013).

${ }^{2}$ M. K. Aktas and T. Ozgumus, "The effects of acoustic streaming on thermal convection in an enclosure with differentially heated horizontal walls,” Int. J. Heat Mass Transf. 53, 5289 (2010).

${ }^{3}$ M. C. Charrier-Mojtabi, A. Fontaine, and A. Mojtabi, "Influence of acoustic streaming on thermo-diffusion in a binary mixture under microgravity," Int. J. Heat Mass Transf. 55, 5992 (2012).

${ }^{4}$ V. Frenkel, R. Gurka, A. Liberzon, U. Shavit, and E. Kimmel, "Preliminary investigations of ultrasound induced acoustic streaming using particle image velocimetry," Ultrasonics 39, 153 (2001).

${ }^{5}$ M. Dhahbi, M. Ben Chiekh, B. Gilles, J. C. Béra, and A. Jemni, "Numerical simulations of particle dynamics in a poststenotic blood vessel region within the scope of extracorporeal ultrasound stenosis treatment," Med. Eng. Phys. 34, $982(2012)$.

${ }^{6}$ D. Gao, Z. Li, Q. Han, and Q. Zhai, "Effect of ultrasonic power on microstructure and mechanical properties of AZ91 alloy," Mater. Sci. Eng. A 502, 2 (2009).

${ }^{7}$ X. Jian, H. Xu, T. T. Meek, and Q. Han, "Effect of power ultrasound on solidification of aluminum A356 alloy," Mater. Lett. 59, 190 (2005).

${ }^{8}$ X. Jian, T. T. Meek, and Q. Han, "Refinement of eutectic silicon phase of aluminum A356 alloy using high-intensity ultrasonic vibration," Scrip. Mater. 54, 893 (2006).

${ }^{9}$ G. N. Kozhemyakin, "Imaging of convection in a Czochralski crucible under ultrasound waves," J. Cryst. Growth 257, 237 (2003).

${ }^{10}$ G. Kozhemyakin, "Influence of ultrasonic vibrations on the growth of InSb crystals," J. Cryst. Growth 149, 266 (1995).

${ }^{11}$ L. Yao, H. Hao, S. Ji, C. Fang, and X. Zhang, "Effects of ultrasonic vibration on solidification structure and properties of Mg-8Li-3Al alloy," Trans. Nonfer. Met. Soc. Chin. 21, 1241 (2011).

${ }^{12} \mathrm{Z}$. Zhang, Q. Le, and J. Cui, "Microstructures and mechanical properties of AZ80 alloy treated by pulsed ultrasonic vibration,” Trans. Nonfer. Met. Soc. Chin. 18, S113 (2008).

${ }^{13}$ H. P. Khadamakar, A. W. Patwardhan, G. Padmakumar, and G. Vaidyanathan, "Flow distribution in the inlet plenum of steam generator,” Nucl. Eng. Des. 241, 4165 (2011). 
${ }^{14}$ H.-C. Nataf, T. Alboussière, D. Brito, P. Cardin, N. Gagnière, D. Jault, and D. Schmitt, "Rapidly rotating spherical Couette flow in a dipolar magnetic field: An experimental study of the mean axisymmetric flow," Phys. Earth Planet. Interiors 170, 60 (2008).

${ }^{15}$ D. Brito, T. Alboussière, P. Cardin, N. Gagnière, D. Jault, P. La Rizza, J.-P. Masson, H.-C. Nataf, and D. Schmitt, "Zonal shear and super-rotation in a magnetized spherical Couette-flow experiment," Phys. Rev. E 83, 066310 (2011).

${ }^{16}$ D. Brito, H.-C. Nataf, P. Cardin, J. Aubert, and J.-P. Masson, "Ultrasonic Doppler velocimetry in liquid gallium,” Exp. Fluids 31, 653 (2001).

${ }^{17}$ A. Pedcenko, A. Bojarevičs, J. Priede, G. Gerbeth, and R. Hermann, "Velocity measurements in the liquid metal flow driven by a two-phase inductor," Exp. Fluids 54, 1558 (2013).

${ }^{18}$ S. Eckert, D. Buchenau, G. Gerbeth, F. Stefani, and F.-P. Weiss, "Some recent developments in the field of measuring techniques and instrumentation for liquid metal flows," J. Nucl. Sci. Technol. 48, 490 (2011).

${ }^{19}$ S. Eckert and G. Gerbeth, "Velocity measurements in liquid sodium by means of ultrasound Doppler velocimetry," Exp. Fluids 32, 542 (2002).

${ }^{20}$ D. Buchenau, S. Eckert, G. Gerbeth, R. Stieglitz, and M. Dierckx, "Measurement technique developments for LBE flows," J. Nucl. Mater. 415, 396 (2011).

${ }^{21}$ T. Schulenberg and R. Stieglitz, "Flow measurement techniques in heavy liquid metals," Nucl. Eng. Des. 240, 2077 (2010).

${ }^{22}$ K. Kikuchi, Y. Takeda, H. Obayashi, M. Tezuka, and H. Sato, "Measurement of LBE flow velocity profile by UDVP ," J. Nucl. Mater. 356, 273 (2006).

${ }^{23}$ A. Pothérat, F. Rubiconi, Y. Charles, and V. Dousset, "Direct and inverse pumping in flows with homogeneous and non-homogeneous swirl," Eur. Phys. J. E 36, 94 (2013).

${ }^{24}$ C. M. Poindexter, P. J. Rusello, and E. A. Variano, "Acoustic Doppler velocimeter-induced acoustic streaming and its implications for measurement," Exp. Fluids 50, 1429 (2011).

${ }^{25}$ S. B. Barnett, G. R. Ter Haar, M. C. Ziskin, H. D. Rott, F. A. Duck, and K. Maeda, "International recommendations and guidelines for the safe use of diagnostic ultrasound in medicine," Ultrasound Med. Biol. 26, 355 (2000).

${ }^{26}$ H. C. Starritt, C. L. Hoad, F. A. Duck, D. K. Nassiri, I. R. Summers, and W. Vennart, "Measurement of acoustic streaming using magnetic resonance," Ultrasound Med. Biol. 26, 321 (2000).

${ }^{27}$ G. Zauhar, H. C. Starritt, and F. A. Duck, "Studies of acoustic streaming in biological fluids with an ultrasound Doppler technique,” Br. J. Radiol. 71, 297 (1998).

${ }^{28}$ G. Zauhar, F. A. Duck, and H. C. Starritt, "Comparison of the acoustic streaming in amniotic fluid and water in medical ultrasonic beams," Ultraschall. Med. 27, 152 (2006).

${ }^{29}$ C. Eckart, "Vortices and streams caused by sound waves," Phys. Rev. 73, 68 (1948).

${ }^{30}$ S. J. Lighthill, “Acoustic streaming," J. Sound Vibr. 61, 391 (1978).

${ }^{31}$ K. D. Frampton, S. E. Martin, and K. Minor, "The scaling of acoustic streaming for application in micro-fluidic devices," Appl. Acoust. 64, 681 (2003).

${ }^{32}$ W. L. Nyborg, Acoustic Streaming (Academic Press, San Diego, 1998).

${ }^{33}$ W. L. Nyborg, "Acoustic streaming near a boundary," J. Acoust. Soc. Am. 30, 329 (1958).

${ }^{34}$ W. Dridi, D. Henry, and H. Ben Hadid, "Stability of buoyant convection in a layer submitted to acoustic streaming," Phys. Rev. E 81, 056309 (2010).

${ }^{35}$ W. L. Nyborg, "Acoustic streaming due to attenuated plane waves," J. Acoust. Soc. Am. 25, 68 (1953).

${ }^{36}$ P. J. Westervelt, "The theory of steady rotational flow generated by a sound field," J. Acoust. Soc. Am. 25, 60 (1953).

${ }^{37}$ T. Kamakura, T. Sudo, K. Matsuda, and Y. Kumamoto, "Time evolution of acoustic streaming from a planar ultrasound source," J. Acoust. Soc. Am. 100, 132 (1996).

${ }^{38}$ V. Botton, W. Dridi, D. Henry, H. Ben Hadid, B. Moudjed, E. Jondeau, and N. Grosjean, "Etude expérimentale et théorique d'un écoulement entrâné par des ultrasons (acoustic streaming)," Actes du XXème Congrès Français de Mécanique, 2011, Sous la direction de David Chapelle, Marc Dahan, Philippe Picart, Collection Hors collection, Diffusion Presses universitaires de Franche-Comté (Presses-ufc, 2012), http://documents.irevues.inist.fr/handle/2042/46539.

${ }^{39}$ A. Boufermel, N. Joly, P. Lotton, M. Amari, and V. Gusev, "Velocity of mass transport to model acoustic streaming: Numerical application to annular resonators," Acta Acust. Unit. Ac. 97, 219 (2011).

${ }^{40} \mathrm{H}$. Mitome, "The mechanism of generation of acoustic streaming," Electron. Commun. Jpn. Pt. III: Fundam. Electron. Sci. 81, 1 (1998).

${ }^{41}$ A. Nowicki, W. Secomski, and J. Wojcik, "Acoustic streaming: Comparison of low-amplitude linear model with streaming velocities measured by 32-Mhz Doppler," Ultrasound Med. Biol. 23, 783 (1997).

${ }^{42}$ A. Nowicki, T. Kowalewski, W. Secomski, and J. Wójcik, "Estimation of acoustical streaming: Theoretical model, Doppler measurements and optical visualisation," Europ. J. Ultrasound 7, 73 (1998).

${ }^{43}$ B. Moudjed, "Caractérisation expérimentale et théorique des écoulements entraînés par ultrasons. Perspectives d'utilisation dans les procédés de solidification du Silicium photovoltaique," Ph.D. thesis (INSA de Lyon, French, 2013).

${ }^{44}$ L. E. Kinsler, A. R. Frey, A. B. Coppens, and J. V. Sanders, Fundamentals of Acoustics (Wiley, 1999).

${ }^{45}$ O. V. Rudenko and A. A. Sukhorukov, "Nonstationary Eckart streaming and pumping of liquid in an ultrasonic field," Acoust. Phys. 44, 565 (1998).

${ }^{46}$ W. Dridi, D. Henry, and H. BenHadid, "Influence of acoustic streaming on the stability of an isothermal or laterally heated fluid layer," C. R. Mec. 335, 175 (2007).

${ }^{47}$ H. Ben Hadid, W. Dridi, V. Botton, B. Moudjed, and D. Henry, "Instabilities in the Rayleigh-Bénard-Eckart problem," Phys. Rev. E 86, 016312 (2012).

${ }^{48}$ W. Dridi, D. Henry, and H. Ben Hadid, "Influence of acoustic streaming on the stability of a laterally heated threedimensional cavity,” Phys. Rev. E 77, 046311 (2008).

${ }^{49}$ W. Dridi, D. Henry, and H. Ben Hadid, "Influence of acoustic streaming on the stability of melt flows in horizontal Bridgman configurations,” J. Cryst. Growth 310, 1546 (2008). 
${ }^{50}$ P. M. Nasch, M. H. Manghnani, and R. A. Secco, "A modified ultrasonic interferometer for sound velocity measurements in molten metals and alloys,” Rev. Sci. Instrum. 65, 682 (1994).

${ }^{51}$ J. M. M. Pinkerton, "The absorption of ultrasonic waves in liquids and its relation to molecular constitution," Proc. Phys. Soc. B 62, 129 (1949).

${ }^{52}$ L. N. Liebermann, "The second viscosity of liquids," Phys. Rev. 75, 1415 (1949).

${ }^{53}$ Kaye \& Laby, "The speed and attenuation of sound," Tables of Physical and Chemical Constants, 16th ed. (Kaye \& Laby, 1995), online version 1.0, 2005, see www.kayelaby.npl.co.uk.

${ }^{54}$ E. Guyon, J.-P. Hulin, L. Petit, and P. G. de Gennes, Hydrodynamique Physique (EDP Sciences Editions, 2001).

${ }^{55}$ E. Guyon, Physical Hydrodynamics (Oxford University Press, 2001).

${ }^{56}$ P. Hariharan, M. R. Myers, R. A. Robinson, S. H. Maruvada, J. Sliwa, and R. K. Banerjee, "Characterization of high intensity focused ultrasound transducers using acoustic streaming," Acoust. Soc. Am. 123, 1706 (2008).

${ }^{57}$ M. R. Myers, P. Hariharan, and R. K. Banerjee, "Direct methods for characterizing high-intensity focused ultrasound transducers using acoustic streaming," J. Acoust. Soc. Am. 124, 1790 (2008).

${ }^{58}$ S. Eckert, G. Gerbeth, and V. I. Melnikov, "Velocity measurements at high temperatures by ultrasound Doppler velocimetry using an acoustic wave guide," Exp. Fluids 35, 381 (2003).

${ }^{59}$ N. Rivière, G. Travin, and R. J. Perkins, "Subcritical open channel flows in four branch intersections," Water Resour. Res. 47, W10517, doi:10.1029/2011WR010504 (2011).

${ }^{60}$ Y. Inatomi, F. Onishi, K. Nagashio, and K. Kuribayashi, "Density and thermal conductivity measurements for silicon melt by electromagnetic levitation under a static magnetic field," Int. J. Thermophys. 28, 44 (2007).

${ }^{61}$ M. Hayashi, H. Yamada, N. Nabeshima, and K. Nagata, "Temperature dependence of the velocity of sound in liquid metals of group XIV,” Int. J. Thermophys. 28, 83 (2007).

${ }^{62}$ W. K. Rhim and K. Ohsaka, "Thermophysical properties measurement of molten silicon by high-temperature electrostatic levitator: Density, volume expansion, specific heat capacity, emissivity, surface tension and viscosity" J. Cryst. Growth 208, 313 (2000).

${ }^{63}$ J. P. Garandet, "New determinations of diffusion coefficients for various dopants in liquid silicon," Int. J. Thermophys. 28, 1285 (2007).

${ }^{64}$ A. S. Okhotin, A. S. Pushkarskii, and V. V. Gorbachev, "Thermophysical properties of semiconductors," Atomizdat, Moscow, 1972.

${ }^{65}$ V. Sobolev, Institutional Repository of the Belgian Nuclear Research Centre (SCK-CEN), "Database of thermophysical properties of liquid metal coolants for GEN-IV," 2nd ed. (SCK•CEN Mol, Belgium, 2011), p. 173 (Open Report of the Belgian Nuclear Research Centre; BLG-1069), see http://publications.sckcen.be/dspace/handle/10038/7739. 